\title{
INTEGRAL CONCENTRATION OF IDEMPOTENT TRIGONOMETRIC POLYNOMIALS WITH GAPS
}

\author{
ALINE BONAMI \& SZILÁRD GY. RÉVÉSZ
}

\begin{abstract}
We prove that for all $p>1 / 2$ there exists a constant $\gamma_{p}>0$ such that, for any symmetric measurable set of positive measure $E \subset \mathbb{T}$ and for any $\gamma<\gamma_{p}$, there is an idempotent trigonometrical polynomial $f$ satisfying $\int_{E}|f|^{p}>\gamma \int_{\mathbb{T}}|f|^{p}$. This disproves a conjecture of Anderson, Ash, Jones, Rider and Saffari, who proved the existence of $\gamma_{p}>0$ for $p>1$ and conjectured that it does not exists for $p=1$.

Furthermore, we prove that one can take $\gamma_{p}=1$ when $p>1$ is not an even integer, and that polynomials $f$ can be chosen with arbitrarily large gaps when $p \neq 2$. This shows striking differences with the case $p=2$, for which the best constant is strictly smaller than $1 / 2$, as it has been known for twenty years, and for which having arbitrarily large gaps with such concentration of the integral is not possible, according to a classical theorem of Wiener.

We find sharper results for $0<p \leq 1$ when we restrict to open sets, or when we enlarge the class of idempotent trigonometric polynomials to all positive definite ones.
\end{abstract}

Mathematics Subject Classification (2000): Primary 42A05. Secondary 42A16, 42A61, 42A55, 42A82, 42B05.

Keywords: idempotent exponential polynomials, $L^{p}$-norm concentration, Hardy-Littlewood majorant problem, Montgomery conjecture, Ingham Inequality, positive definite trigonometric polynomials, inhomogeneous Diophantine approximation, Marcinkiewicz-Zygmund Inequality, Bernstein's Inequality, random trigonometric polynomials.

Date: November 19, 2018.

The second author was supported in part by the Hungarian National Foundation for Scientific Research, Project \#s T-049301 T-049693 K-61908 and K-72731.

This work was accomplished during the second author's stay at the Institut Henri Poincaré in Paris under his Marie Curie fellowship, contract \# MEIF-CT2005-022927. 


\section{INTRODUCTION}

In this work $\mathbb{T}:=\mathbb{R} / \mathbb{Z}$ is the circle, and $e(t):=e^{2 \pi i t}$ is the usual exponential function adjusted to interval length 1 . We will denote $e_{h}$ the function $e(h x)$. For obvious reasons of being convolution idempotents, the set

$$
\mathcal{P}:=\left\{\sum_{h \in H} e_{h}: H \subset \mathbb{N}, \# H<\infty\right\}
$$

is called the set of (convolution-)idempotent exponential (or trigonometric) polynomials, or just idempotents for short.

Remark that we assume all frequencies of idempotents under consideration to be non-negative. We can do without loss of generality since we will only be interested in the modulus of idempotents, which is not modified by multiplication by some exponential $e_{N}$. We will denote as well

$$
\mathcal{T}:=\left\{\sum_{h \in H} a_{h} e_{h}: H \subset \mathbb{N}, \# H<\infty ; a_{h} \in \mathbb{C}, h \in H\right\}
$$

the space of all trigonometric polynomials.

The starting point of our work was a conjecture in [3] regarding the impossibility of the concentration of the integral norm of idempotents.

Before recording the main result of the paper [3], let us give some notations and definitions. We first start by the notion of concentration on symmetric open sets, for which results are more complete, and proofs are more elementary.

A set $E$ is symmetric if $x \in E$ implies $-x \in E$.

Definition 1. Let $p>0$ and $a \in \mathbb{T}$. We say that there is $p$-concentration at $a$ if there exists a constant $c>0$ so that for any symmetric open set $E$ that contains a, one can find an idempotent $f \in \mathcal{P}$ with

$$
\int_{E}|f|^{p} \geq c \int_{\mathbb{T}}|f|^{p}
$$

Moreover, the supremum of all such constants $c$ will be denoted as $c_{p}(a)$ : it is called the level of the $p$-concentration at $a$. Such an idempotent $f$ will be called a $p$-concentrating polynomial.

Definition 2. Let $p>0$. We say that there is $p$-concentration if there exists a constant $c>0$ so that for any symmetric non empty open set $E$ one can find an idempotent $f \in \mathcal{P}$ with

$$
\int_{E}|f|^{p} \geq c \int_{\mathbb{T}}|f|^{p}
$$


Moreover, $c_{p}$ will denote the supremum of all such constants c. Correspondingly, $c_{p}$ is called the level of $p$-concentration. If $c_{p}=1$, we say that there is full $p$-concentration.

Clearly, as remarked in [13], the local constant $c_{p}(a)$ is an upper semi-continuous function on $\mathbb{T}$, and $c_{p}=\inf _{a \in \mathbb{T}} c_{p}(a)$.

Remark 3. We have taken symmetric open sets because the function $|f|$ is even for $f \in \mathcal{P}$. Without the assumption of symmetry, the constant $c_{p}(a)$ would be at most $1 / 2$ for a different from 0 and $1 / 2$. With this definition, as we will see, $c_{p}(a)$ and even $c_{p}$ can achieve the maximal value 1. Nevertheless, using the alternative definition with arbitrary open sets (or just intervals) would only mean taking half of our constants $c_{p}(a)$ for $a \neq 0,1 / 2$ and of $c_{p}$.

The question of $p$-concentration, and the computation or at least estimation of the best constant $c_{p}$, originated from the work of Cowling [12], and of Ash [4] on comparison of restricted type and strong type for convolution operators. This is described recently in the survey [5]. It has since then been the object of considerable interest, with improving lower bounds obtained by Pichorides, Montgomery, Kahane and Ash, Jones and Saffari, see [1, 2, 3] for details. In 1983 DéchampsGondim, Piquard-Lust and Queffélec [13, 14] answered a question from [1], proving the precise value

$$
c_{2}=\sup _{0 \leq x} \frac{2 \sin ^{2} x}{\pi x}=0.46 \cdots .
$$

Moreover, they obtained $c_{p} \geq 2^{1-\frac{p}{2}} c_{2}^{p / 2}$ for all $p>2$.

As in [13, 14, 2, 3], we will consider the same notion of $p$-concentration of (convolution-)idempotents for measurable sets, too.

Definition 4. Let $p>0$ and $a \in \mathbb{T}$. We say that there is $p$-concentration for measurable sets at $a$, if there exists a constant $\gamma>0$ so that for any symmetric measurable set $E$, with a being a density point of $E$, there exists some idempotent $f \in \mathcal{P}$ with

$$
\int_{E}|f|^{p} \geq \gamma \int_{\mathbb{T}}|f|^{p} .
$$

The supremum of all such constants $\gamma$ will be denoted as $\gamma_{p}(a)$. Furthermore, we say that there is p-concentration for measurable sets if such an inequality holds for any symmetric measurable set $E$ of positive measure. The supremum of all such constants is denoted by $\gamma_{p}$.

It is clear that $p$-concentration for measurable sets implies $p$-concentration. On the other hand it is not clear, if $\gamma_{p}(a)$ is upper semicontinuous, too. If we knew this, by our methods that would easily imply the same strength of the results for measurable sets, as we will obtain for open sets. 
The main theorem of [3] can be stated as:

Theorem 5 (Anderson, Ash, Jones, Rider, Saffari). There is $p$-concentration for measurable sets for all $p>1$.

We also refer to them for the fact that $\gamma_{2}=c_{2}$ is given by (5). The proof of [2, 3] is based on the properties of the function

$$
D_{n}(x) D_{n}(q x) \text {, }
$$

where $D_{n}$ stands for the Dirichlet kernel. We will use the same notation as in [3] and define the Dirichlet kernel as

$$
D_{n}(x):=\sum_{\nu=0}^{n-1} e(\nu x)=e^{\pi i(n-1) x} \frac{\sin (\pi n x)}{\sin (\pi x)} .
$$

The idea is that the first Dirichlet kernel in (77) will have sufficiently peaky behavior (regarding $|\cdot|^{p}$ ), while the second one simulates a Dirac delta, so that the $p$-th integral outside very close neighborhoods of the points $k / q$ is small. They use the multiplicative group structure of $\mathbb{Z} / q \mathbb{Z}$, when $q$ is prime, to prove that concentration at $k / q$ and concentration at $1 / q$ may be compared.

Their proof yields $p$-concentration only with $c_{p} \rightarrow 0$ when $p \rightarrow 1$. Based on these and some other heuristical arguments and calculations the authors conjectured that for $p$-concentration the value 1 should be a natural limit. We will disprove this conjecture, even for measurable sets and we will even prove more: all concentrating idempotents can be taken with arbitrary large gaps. Recall that the trigonometric polynomial

$$
f(x):=\sum_{k=1}^{K} a_{k} e\left(n_{k} x\right),
$$

has gaps larger than $N$ if it satisfies the gap condition $n_{k+1}-n_{k}>N$ $(k=1, \ldots, K-1)$. Before describing our results more precisely, we need other definitions.

Definition 6. We say that there is p-concentration with gap (resp. pconcentration with gap for measurable sets) at a if for all $N>0$ the p-concentrating polynomial in (3) (resp. in (6) ) can be chosen with gap larger than $N$. If this holds for every a, we say that there is $p$ concentration with gap (resp. p-concentration with gap for measurable sets). If, moreover, the constant c can be taken arbitrarily close to 1 , we say that there is full p-concentration with gap (resp. p-concentration with gap for measurable sets).

With these definitions, we can give our main theorems.

Theorem 7. For all $0<p<\infty$ we have $p$-concentration. Moreover, if $p$ is not an even integer, then we have full concentration, i.e. $c_{p}=1$. 
When considering even integers, we have $c_{2}$ given by (5), then $0.495<$ $c_{4} \leq 1 / 2$, then for all other even integers $0.483<c_{2 k} \leq 1 / 2$. Moreover, unless $p=2$, we have concentration with gap at the same level of concentration. On the other hand for $p=2$ requiring arbitrarily large gaps would decrease the level of concentration to 0 .

For measurable sets, our results are just as good for $p>1$. Arriving at the limits of our current methods, we leave it as an open problem what happens for $p \leq 1 / 2$, and whether there is full concentration for $1 / 2<p \leq 1$.

Theorem 8. For all $1 / 2<p<\infty$ we have $p$-concentration for measurable sets. If $p$ is not an even integer, then we have full concentration for measurable sets when $p>1$. If $p=2$, the level of the concentration is given by (5), and for $p=4$ we have $0.495<\gamma_{4} \leq 1 / 2$. For other even integers we have uniformly $0.483<\gamma_{2 k} \leq 1 / 2$. Moreover, unless $p=2$, the same level of concentration can be achieved with arbitrarily large gaps.

This improves considerably the constants given in [2, 3], which tend to zero when $p \rightarrow \infty$ or when $p \rightarrow 1^{+}$(however, to compare constants, be aware of the notational difference between us and [3, 2]).

We postpone to the last part of the paper what concerns measurable sets. The proofs will follow from an adaptation of the methods that we develop for open sets, and also from the use of diophantine approximation. As in [3], we do not know whether constants $\gamma_{p}$ and $c_{p}$ differ when $p \neq 2$, except when we know that both of them are 1 , which is the case of all $p>1$ not an even integer.

Let us hint some of the key ideas in our proofs, which may be of independent interest. The first one is an explicit construction of concentrating idempotents for the points 0 and $1 / 2$ at a level of concentration arbitrarily close to 1 and with arbitrarily large gaps. To emphasize their role in our construction, we will term such concentrating idempotents as "peaking idempotents", or, when referring to the large gaps required, as "gap-peaking idempotents" - for a more precise meaning see the beginning of $\$ 3$.

Proposition 9. For all $p>0$, except for $p=2$, one has full $p$ concentration with gap at 0 . For $p=2$, positive concentration with arbitrarily large gaps is possible at neither points $a \in \mathbb{T}$.

Note that, using the Dirichlet kernel that peaks at 0 , we find full $p$ concentration at 0 for $p>1$. For $p \leq 1$, the Dirichlet kernel cannot be used. For a given concentration, our examples will be obtained using idempotents of much higher degree. So as for the behavior at point 0 and $p>1$ different from 2 , the novelty is the fact that the peaking polynomial may have arbitrarily large gaps. 
This is what cannot occur in $L^{2}$, in view of Ingham's inequalities [19, 34]. The somewhat surprising new fact here is that it does occur for all other values of $p$.

Zygmund [34, Chapter V $\S 9$, page 380] pointed out concerning Ingham's results on essentially uniform distribution of square integrals (norms) for Fourier series with large gaps: "Nothing seems to be known about possible extensions to classes $L^{p}, p \neq 2$ ". To the best of our knowledge the problem has not been addressed thus far. But now we find that an Ingham type inequality is characteristic to the Hilbertian case, and for no $p \neq 2$ one can have similar inequalities, not even when restricting to idempotent polynomials.

The next proposition is even more surprising. It is the key to full concentration at other points than 0 .

Proposition 10. Full p-concentration with gap at $1 / 2$ holds whenever $p>0$ is not an even integer. On the other hand, for $p=2 k \in 2 \mathbb{N}$, $c_{2 k}(1 / 2)=1 / 2$.

The assertion for $p$ an even integer will follow directly from the work of Déchamps-Gondim, Lust-Piquard and Queffélec [13, 14].

For $0<p<2$ we base our argument on the properties of the bivariate idempotent $1+e(y)+e(x+2 y)$.

For $p>2$, we will rely on a construction of Mockenhaupt and Schlag, see 23 , given in their work on the Hardy-Littlewood majorant problem, which we describe now in its original formulation. Following Hardy and Litlewood, $f$ is said to be a majorant to $g$ if $|\widehat{g}| \leq \widehat{f}$. Obviously, then $f$ is necessarily a positive definite function. The (upper) majorization property (with constant 1 ) is the statement that whenever $f \in L^{p}(\mathbb{T})$ is a majorant of $g \in L^{p}(\mathbb{T})$, then $\|g\|_{p} \leq\|f\|_{p}$. Hardy and Littlewood proved this for all $p \in 2 \mathbb{N}$. On the other hand already Hardy and Littlewood observed that this fails for $p=3$ : they took $f=1+e_{1}+e_{3}$ and $g=1-e_{1}+e_{3}$ (where $e_{k}(x):=e(k x)$ ) and calculated that $\|f\|_{3}<\|g\|_{3}$.

The failure of the majorization property for $p \notin 2 \mathbb{N}$ was shown by Boas [8] (see also [7] for arbitrarily large constants, and also [16, 22] for further comments and similar results in other groups.) Montgomery conjectured that it fails also if we restrict to majorants belonging to $\mathcal{P}$, see [24, p. 144]. This has been recently proved by Mockenhaupt and Schlag in [23].

Theorem 11 (Mockenhaupt \& Schlag). Let $p>2$ and $p \notin 2 \mathbb{N}$, and let $k>p / 2$ be arbitrary. Then for the trigonometric polynomials $g:=\left(1+e_{k}\right)\left(1-e_{k+1}\right)$ and $f:=\left(1+e_{k}\right)\left(1+e_{k+1}\right)$ we have $\|g\|_{p}>\|f\|_{p}$.

Our proof of Proposition 10 for $p>2$ and $p \notin 2 \mathbb{N}$, will be based on the construction of Mockenhaupt and Schlag.

Once we have our peaking polynomials at $1 / 2$, we conclude in proving the following assertion. 
Proposition 12. Let $p>0$ and assume that we have full $p$-concentration with gap at $1 / 2$ for this value of $p$. Then we also have p-concentration. Moreover, $c_{p}=1$ and we have full p-concentration with gap.

The proof of Proposition 12 consists of considering products like

$$
D_{r}\left(s_{1} x\right) \cdots D_{r}\left(s_{n} x\right) T(q x),
$$

where the similarity to (7) may be misleading in regard of the role of the Dirichlet kernels here: the role of the "approximate Dirac delta" is fully placed on $T$, which is a peaking function at $1 / 2$ with large gaps that insure that the product is still an idempotent. The first factors will be chosen in such a way that they coincide with a power of a Dirichlet kernel on some grid $\frac{1}{2 q}+\mathbb{Z} / q \mathbb{Z}$. For measurable sets, the use of diophantine approximation forces us to take at most two factors, resulting in the restriction $p>1 / 2$.

When there is not full $p$-concentration at $1 / 2$, i.e. for $p=2 k$, we could not determine $c_{2 k}$ precisely. Still, we can use a peaking function at 0 , provided by Proposition 9, thus obtaining reasonable uniform bounds.

Our last results derive from the consideration of the class of positive definite trigonometric polynomials

$$
\mathcal{P}^{+}:=\left\{\sum_{h \in H} a_{h} e_{h}: H \subset \mathbb{N}, \# H<\infty ; a_{h}>0 \text { for } h \in H\right\},
$$

for which full $p$-concentration for measurable sets can be proved for $p>0$ not an even integer. We then use a randomization process to transfer this result to the class $\mathcal{P}$ for $p>2$, and then using that even to $p>1$.

Let us record here two remarks on further developments of the results given in the present paper.

Remark 13. The above results are well adapted to give counter-examples for the Wiener property, which is concerned with the possibility of inferring $f \in L^{p}(\mathbb{T})$ for positive definite functions $f$ having large gaps in case we know $f \in L^{p}(I)$ on some small interval (or even measurable set). For developments in this direction see [9]. For previous counterexamples to the Wiener property for $p>2$, see the references cited in [9], and also the constructions given by Erdös and Rényi [15] with an existential proof, and, for $p>6$, by Turán [32] with a concrete construction.

Remark 14. As seen above, the conjecture of Ash, Anderson, Jones, Rider and Saffari on nonexistence of $L^{1}$-concentration, described after Theorem 5, fails. But in a sense this is due to a "cheating" in the extent that we can simulate powers of Dirichlet kernels by products of their scaled versions. In a forthcoming note [10] we show, however, that on the finite groups $\mathbb{Z} / q \mathbb{Z}$ uniform in $q L^{1}$ concentration does really fail. 
Let us finally fix some notations that will be used all over. We denote

$$
\mathcal{T}_{q}:=\left\{\sum_{h=0}^{q-1} a_{h} e_{h} ; a_{h} \in \mathbb{C} \text { for } h=0, \cdots, q-1\right\}
$$

the space of trigonometric polynomials of degree smaller than $q$ and

$$
\mathcal{P}_{q}:=\left\{\sum_{h \in H} e_{h}: H \subset\{0,1, \cdots q-1\}\right\}
$$

the set of idempotents of degree smaller than $q$.

Aknowledgement. The authors thank Terence Tao, who suggested the construction of peaking functions through bivariate idempotents and Riesz products [29]. Although Riesz products form a well-known technique, see e.g. [7, 17, 22, and bivariate idempotents have already been occurred in the subject, too, see [2, 3], combining these for the particular construction did not occur to us, so the present paper could not have been written without this contribution.

The authors thank also Gerd Mockenhaupt and Wilhem Schlag for giving them their recent manuscript on the Hardy and Littlewood majorant problem [23]. One of their construction plays a crucial role in this paper. 


\section{Part I: Limitations of full concentration.}

\section{Negative Results REgarding CONCENTRAtion When $p \in 2 \mathbb{N}$}

Let us first start with proving that in case $p=2$, requiring arbitrarily large gaps decreases the level of concentration to 0 , as said in Theorem 7 and Proposition 9 (and, consequently, in Theorem 8, too).

For this there is a well known argument. We take an interval $E$ centered at 0 and a triangular function $\Delta$ supported by $2 E$ and equal to 1 at zero. Let $N$ be an integer and $f$ an idempotent with gap $N$. Then

$$
\int_{E}|f|^{2} d t \leq 2 \int \Delta|f|^{2} d t=2 \sum_{m} \sum_{n} \widehat{\Delta}(m) \widehat{f}(n) \overline{\widehat{f}(n+m)} .
$$

If we write separately the term with $m=0$ and insert $\widehat{\Delta}(0)=|E|$, then the right hand side becomes

$$
2|E| \sum_{n}|\widehat{f}(n)|^{2}+2 \sum_{|m|>N} \widehat{\Delta}(m) \sum_{n} \widehat{f}(n) \overline{\widehat{f}(n+m)} .
$$

Finally, by an application of the Cauchy-Schwarz inequality,

$$
\int_{E}|f|^{2} d t \leq 2|E| \sum_{n}|\widehat{f}(n)|^{2}+2 \sum_{|m|>N}|\widehat{\Delta}(m)| \sum_{n}|\widehat{f}(n)|^{2} .
$$

According to Parseval's identity $\int_{\mathbb{T}}|f|^{2} d t=\sum_{n}|\hat{f}(n)|^{2}$, hence

$$
\frac{\int_{E}|f|^{2} d t}{\int_{\mathbb{T}}|f|^{2} d t} \leq 2|E|+2 \sum_{|m|>N}|\widehat{\Delta}(m)| .
$$

The last estimate can be taken arbitrarily small by taking the interval $E$ small enough, and then the gap $N$ large enough, using the fact that the Fourier series of $\Delta$ is absolutely convergent. This contradicts the peaking property with gap.

Remark 15. The same proof, using for $\Delta$ a triangular function supported by $E$, gives the reverse inequality

$$
\frac{\int_{E}|f|^{2} d t}{\int_{\mathbb{T}}|f|^{2} d t} \geq \frac{|E|}{2+\epsilon},
$$

valid for functions with sufficiently large gaps, depending on $E$ and $\epsilon>$ 0. These type of estimates are known as Ingham type inequalities, and various generalizations have many applications e.g. in control theory, see [21], [30], 31]. The fact that one can have full p-concentration with gap at 0 may be interpreted as the impossibility of an Ingham type inequality for $p \neq 2$. This settles to the negative a problem posed by Zygmund, see Notes to Chapter V§9, page 380 in [34]. 
Next, we explain how to obtain the necessary condition $c_{2 k} \leq 1 / 2$. In fact one knows more, since this is also valid for the problem of concentration on the class $\mathcal{P}^{+}$of positive definite exponential polynomials (see (11) ). Let us denote by $c_{p}^{+}$and $c_{p}(a)^{+}$, as well as $\gamma_{p}^{+}$and $\gamma_{p}^{+}(a)$, the corresponding concentration constants, with the class $\mathcal{P}$ of idempotents replaced by the class $\mathcal{P}^{+}$. One has the inequalities

$$
c_{p}(a) \leq c_{p}^{+}(a), \quad c_{p} \leq c_{p}^{+}, \quad \gamma_{p}(a) \leq \gamma_{p}^{+}(a) \quad \gamma_{p} \leq \gamma_{p}^{+} .
$$

It was proved in [13, 14] that $c_{2}^{+}(1 / 2)=1 / 2$. From this we obtain that for $p=2 k$ an even integer, $c_{2 k}(1 / 2) \leq c_{2 k}^{+}(1 / 2) \leq 1 / 2$. Indeed, if $f \in \mathcal{P}^{+}$, so is $f^{k}$, and using the already known value $c_{2}^{+}(1 / 2)=1 / 2$ we infer $c_{2 k}(1 / 2) \leq c_{2}^{+}(1 / 2)=1 / 2$. In fact we have equality,

$$
c_{2 k}(1 / 2)=c_{2 k}^{+}(1 / 2)=1 / 2,
$$

taking the Dirichlet kernel $D_{N}(2 x)$ as concentrating polynomial.

While [13, 14] gives also $c_{2}^{+}=1 / 2$, we do not know the exact values of $c_{2 k}$ and $c_{2 k}^{+}$for $k>1$.

We do not have any other negative result than the ones in this $\S$.

\section{Part II: Concentration on open sets.}

\section{Full CONCEntration With Gap AND PEAKing FunCtions}

In this section, we will prove Proposition 9 and Proposition 10, For $a=0$ or $1 / 2$, we are interested in the construction of gap-peaking idempotents, that is, for all $\varepsilon, \delta$ and $N>0$, idempotent exponential polynomials

$$
T(x):=\sum_{k=1}^{K} e\left(n_{k} x\right),
$$

with gap condition $n_{k+1}-n_{k}>N(k=1, \ldots, K)$, so that

$$
\int_{a-\delta}^{a+\delta}|T|^{p}>(1-\varepsilon) \int_{\mathbb{T}}|T|^{p} .
$$

The first step is to prove the following.

Proposition 16. Let $f$ be an idempotent exponential polynomial in two variables and of the form

$$
f(x, y)=\sum_{k=1}^{K} e\left(n_{k} x+m_{k} y\right),
$$

where $K \in \mathbb{N}$ and $n_{k}, m_{k} \in \mathbb{N}$ are two sequences of nonnegative integers, with $m_{k}$ strictly increasing. Assume that $f$ has the property that its 
"marginal p-integral", given by

$$
F(x):=\int_{0}^{1}|f(x, y)|^{p} d y
$$

has a strict maximum at $a$, for $a=0$ or $a=1 / 2$. Then one has full p-concentration with gap at the point a.

Proof. Choose $M$ with $0 \leq m_{k}, n_{k}<M$ for all $k$ and consider the Riesz product

$$
g(x):=g_{R, J}(x):=\prod_{j=1}^{J} f\left(x, R^{j} x\right)
$$

where $R$ is a very large integer, $f$ is given by (16) satisfying the assumption, and $J$ will be chosen later on. If we take $R>M(J+1)$, then $g \in \mathcal{P}$; moreover, $g$ will obey a gap condition of size $N$ if $R$ is large enough depending on $J, M$ and $N$. Recall that the marginal $p$-integral (17) has a strict maximum at $a$. For any fixed interval $I$, the integral of $|g|^{p}$ on $I$ will approach the integral of $F^{J}$ on $I$ as $R \rightarrow \infty$. Indeed,

$$
\int_{I}|g|^{p}=\int_{I} \prod_{j=1}^{J}\left|f\left(x, R^{j} x\right)\right|^{p} d x
$$

and as the function $|f|^{p} \in C\left(\mathbb{T}^{2}\right)$, we can apply Lemma 17] below.

Lemma 17. Assume that $\varphi \in C\left(\mathbb{T} \times \mathbb{T}^{J}\right)$. Denote the marginal integrals by $\Phi(x):=\int_{\mathbb{T}^{J}} \varphi(x, \mathbf{y}) d \mathbf{y}$. Then, for $E$ a measurable set of positive measure, we have

$$
\lim _{n_{1}, n_{2}, \ldots, n_{J} \rightarrow \infty} \int_{E} \varphi\left(x, n_{1} x, n_{1} n_{2} x, \ldots, n_{1} n_{2} \cdots n_{J} x\right) d x=\int_{E} \Phi(x) d x .
$$

Here by $n_{1}, \ldots, n_{J} \rightarrow \infty$ we naturally mean $\min \left(n_{1}, \ldots, n_{J}\right) \rightarrow \infty$. For the sake of remaining self-contained, we give a proof below, even if this one is standard, mentioned also e.g. in [22, 24, 7] (for $J=1$ ).

Proof. By density, it is sufficient to prove this for $\varphi$ an exponential polynomial on $\mathbb{T} \times \mathbb{T}^{J}$. By linearity, it is sufficient to consider a monomial. When it does not depend on the second variable there is nothing to prove. Assume that $\varphi(x, \mathbf{y})=e\left(k x+l_{1} y_{1}+\cdots+l_{J} y_{J}\right)$, with at least one of the $l_{j}$ 's being nonzero. We want to prove that

$$
\int_{E} \varphi\left(x, n_{1} x, n_{1} n_{2} x, \ldots, n_{1} n_{2} \cdots n_{J} x\right) d x \longrightarrow 0 \quad\left(n_{1}, \ldots, n_{J} \rightarrow \infty\right) .
$$

This integral is the Fourier coefficient of the characteristic function of $E$ at the frequency $k+n_{1} l_{1}+n_{1} n_{2} l_{2}+\cdots+n_{1} n_{2} \cdots n_{J} l_{J}$, which tends to 
infinity for $n_{1}, \ldots, n_{J} \rightarrow \infty$. We conclude using the Riemann-Lebesgue Lemma.

Let us go back to our Riesz product $g$ in (18). Let us first choose $J$ large enough: Then $F^{J}$ will be arbitrarily concentrated on $I:=$ $[a-\delta, a+\delta]$ in integral because $F$ has a strict global maximum at $a$. More precisely, we fix $J$ large enough so that

$$
\int_{I} F^{J}>(1-\varepsilon) \int_{\mathbb{T}} F^{J} .
$$

Once $J$ is fixed, we use Lemma 17 for the function

$$
\varphi(x, \mathbf{y}):=\prod_{j=1}^{J}\left|f\left(x, y_{j}\right)\right|^{p} .
$$

We know that

$$
\lim _{R \rightarrow \infty} \int_{I}\left|g_{R, J}\right|^{p}=\int_{I} F^{J},
$$

and the same for the integral over the whole torus. The proposition is proved.

This concludes the proof of Proposition 9, assuming that the condition of Proposition 16 holds. Next we will focus on this point.

Remark 18. The function $|f|$ is even in the sense that $|f(-x,-y)|=$ $|f(x, y)|$, since the quantities inside the absolute value sign are just complex conjugates. Therefore, $F$ is even. Moreover it can have a unique maximum in $\mathbb{T}$ if only this maximum is either at 0 or at $1 / 2$.

Proposition 19. Let $f(x, y):=1+e(y)+e(x+2 y)$. Then the marginal integral function $F_{p}(x):=\int_{0}^{1}|f(x, y)|^{p} d y$ is a continuous function, which has a unique, strict maximum at 0 for $p>2$, while it has a strict maximum at $1 / 2$ for $p<2$.

Proof. Since $F_{p}$ is even, it suffices to prove that it is monotonic on $\left[0, \frac{1}{2}\right]$, with the required monotonicity. Note that

$$
|f(x, y)|=|2 e(x / 2) \cos (\pi(x+2 y))+1| .
$$

So

$$
\begin{aligned}
F_{p}(x) & =\int_{-1 / 2}^{1 / 2}|2 e(x / 2) \cos (2 \pi y)+1|^{p} d y \\
& =\int_{-1 / 4}^{1 / 4}\left(|2 e(x / 2) \cos (2 \pi y)+1|^{p}+|2 e(x / 2) \cos (2 \pi y)-1|^{p}\right) d y .
\end{aligned}
$$


It is sufficient to show that for fixed $y \in\left(-\frac{1}{4}, \frac{1}{4}\right)$ the quantity

$$
\Phi(x, y):=|2 e(x / 2) \cos (2 \pi y)+1|^{p}+|2 e(x / 2) \cos (2 \pi y)-1|^{p}
$$

is monotonic in $x$ for $0<x<\frac{1}{2}$. Considering its derivative

$$
\begin{aligned}
\frac{\partial \Phi}{\partial x}(x, y)= & -2 p \pi \sin (\pi x) \cos (2 \pi y) \\
& \times\left\{\left|2 e\left(\frac{x}{2}\right) \cos (2 \pi y)+1\right|^{p-2}-\left|2 e\left(\frac{x}{2}\right) \cos (2 \pi y)-1\right|^{p-2}\right\}
\end{aligned}
$$

we find that its signum is the opposite of the signum of the difference in the second line. It follows that $\Phi$, hence $F_{p}$ has a strict global maximum at zero when $p>2$ and a strict global maximum at $1 / 2$ when $p<2$.

This concludes for the existence of a peaking function at 0 for $p>2$, and for a peaking function at $1 / 2$ for $p<2$.

We will need the following lemma later on.

Lemma 20. The function $F_{p}$ is a $\mathcal{C}^{2}$ function for $p>2$ and its second derivative at 0 is strictly negative. For all values of $p$ it is a $\mathcal{C}^{\infty}$ function outside 0 . Its second derivative at $1 / 2$ is strictly negative for $p<2$.

Proof. For $p>2$ the smoothness of the composite function follows from smoothness of $|\cdot|^{p}$. We already know from monotonicity of $\Phi(x, y)$ for fixed $y$ that $\Phi_{x x}^{\prime \prime}(0, y)$ is non positive. Since it is clearly not identically 0 , it is somewhere strictly negative, hence $F_{p}^{\prime \prime}(0)<0$. To prove that $F_{p}$ is a $\mathcal{C}^{\infty}$ function outside 0 , it is sufficient to remark that $f(x, y)$ does not vanish for $x \neq 0$. The same reasoning as above gives the sign of the second derivative at $1 / 2$.

Proof of Proposition 10. Let us now concentrate on peaking functions at $1 / 2$ for $p>2$ not an even integer and prove Proposition 10, We will prove the following, which relies entirely on the methods of Mockenhaupt and Schlag [23], but tailored to our needs with introducing also a second variable and slightly changing the occurring idempotents, too.

Proposition 21. Let $p>2$ not an even integer. For $k$ an odd number that is larger than $p / 2$, the bivariate idempotent function

$$
g(x, y):=\left(1+e_{1}(x) e_{k}(y)\right)\left(1+e_{1}(x) e_{k+1}(y)\right)
$$

is such that its marginal integral $G_{p}(x):=\int_{\mathbb{T}}|g(x, y)|^{p} d y$ has a strict maximum at $1 / 2$. Moreover, it is a $\mathcal{C}^{4}$ function, whose second derivative at $1 / 2$ is strictly negative.

Proof. After a change of variables, we see that

$$
G_{p}(x)=4^{p} \int_{0}^{1}|\cos (\pi k y)|^{p}\left|\cos \left(\pi(k+1)\left(y-\frac{x}{k(k+1)}\right)\right)\right|^{p} d y .
$$


The smoothness of $G_{p}$ follows from the fact that it is the convolution of two functions of class $\mathcal{C}^{2}$. Mockenhaupt and Schlag have computed that

$$
2^{p}|\cos (\pi y)|^{p}=\sum_{n}(-1)^{n} c_{n} e^{2 i \pi n y}
$$

with real coefficients $c_{n}=c_{-n}$, such that, for non negative $n$,

$$
c_{n+1}=\frac{n-\frac{p}{2}}{n+\frac{p}{2}+1} c_{n} .
$$

In the convolution, only frequencies that are multiples of both $k$ and $k+1$ are present, so that

$$
G_{p}(x)=\sum_{n}(-1)^{n} c_{k n} c_{(k+1) n} e^{2 i \pi n x} .
$$

Indeed, the Fourier coefficient $\widehat{G_{p}}(n)$ is equal to $c_{m} c_{m^{\prime}}$, where $k m=$ $(k+1) m^{\prime}$, and $n=m^{\prime} / k$, which gives also $m=(k+1) n$.

Now, looking at the inductive formula for the coefficients, and using the fact that all $c_{k n} c_{(k+1) n}$ are positive for $k>p / 2$, we find that $G_{p}$ is maximum when $e^{2 i \pi n x}=(-1)^{n}$ for all $n$, that is, for $x=1 / 2$. The computation of the Fourier series of its second derivative implies that it is strictly negative at this point.

It remains to prove that we have the gap peaking property at 0 for $0<p<2$. It could be deduced from the theorems below, but we can also build on the construction of Mockhenhaupt and Schlag. Indeed, consider for $0<p<2$, the bivariate idempotent

$$
h(x, y):=\left(1+e_{1}(y)\right)\left(1+e_{1}(x) e_{3}(y)\right) .
$$

Using the computations of Mockenhaupt and Schlag, similarly to the above it is again straightforward to see that the $p$-th marginal integral $H_{p}(x):=\int_{\mathbb{T}}|h(x, y)|^{p} d y$ has a strict maximum at 0 .

This concludes the proof of Proposition 10 .

\section{Restriction to A DiscRete PROBlem of CONCENTRATION}

The second step of our proof consists of restricting the problem of $p$-concentration of an idempotent polynomial on a small interval into the one of concentration of an idempotent polynomial at one point of either of the two discrete grids

$$
\mathbb{G}_{q}:=\frac{1}{q} \mathbb{Z} / q \mathbb{Z} \quad \mathbb{G}_{q}^{\star}:=\frac{1}{2 q}+\frac{1}{q} \mathbb{Z} / q \mathbb{Z} .
$$

The idea is that if we take a gap-peaking polynomial $T$, then multiplication by $T(q x)$ will concentrate integrals on a neighborhood of the grid: for the first grid we need $T$ to be peaking at 0 , and for the second one we need $T$ to do so at $1 / 2$. 
Definition 22. For $f \in \mathcal{T}$ we denote by $\boldsymbol{\Pi}_{q}(f)$ the polynomial in $\mathcal{T}_{q}$ which coincides with $f$ on the grid $\mathbb{G}_{q}$, that is, the polynomial having Fourier coefficients

$$
\widehat{\Pi_{q}(f)}(k):=\sum_{j \in \mathbb{N}} \widehat{f}(k+j q), \quad k=0,1, \cdots, q-1 .
$$

In particular, if $f$ is positive definite, so is $\boldsymbol{\Pi}_{q}(f)$. However, in general the class of idempotent polynomials is not preserved by this projection.

Let us first define concentration on $\mathbb{G}_{q}$.

Definition 23. We shall say that there is $p$-concentration at $a / q$ on $\mathbb{G}_{q}$ with constant $c>0$ if there exists an idempotent polynomial $R$ such that

$$
\left|R\left(\frac{a}{q}\right)\right|^{p}>c \sum_{k=0}^{q-1}\left|R\left(\frac{k}{q}\right)\right|^{p} .
$$

The next well-known lemma (see [13, 3] etc.) allows to restrict to $a=1$.

Lemma 24. Assume that there is p-concentration at $1 / q$ on $\mathbb{G}_{q}$ with constant $c$, that is, with some appropriate idempotent $R$ we have

$$
\left|R\left(\frac{1}{q}\right)\right|^{p}>c \sum_{k=0}^{q-1}\left|R\left(\frac{k}{q}\right)\right|^{p} .
$$

Let now $a \in \mathbb{N}, 0<a<q$ be a natural number so that $a$ and $q$ are relatively prime. Then there is also p-concentration at $a / q$ on $\mathbb{G}_{q}$ with constant $c$ : that is, (23) implies (22) with some appropriately chosen (possibly different) idempotent $R$.

Proof. Let $Q$ be the idempotent that satisfies (23). Let now $a \not \equiv 0,1$ $(\bmod q$, of course) be another value, coprime to $q$. We then have a multiplicative inverse $b$ of $a \bmod q$ so that $1 \leq b<q$ and $a b \equiv 1 \bmod$ $q$. With this particular $b$ we can consider

$$
R(x):=Q(b x) .
$$

Clearly we have $R(0)=Q(0), R(a / q)=Q(a b / q)=Q(1 / q)$, and the values of $R(j / q)=Q(j b / q)$ with $j=0, \ldots, q-1$ will cover all values of $Q(k / q)$ with $k=0,1, \ldots, q-1$, exactly once each. Therefore, we conclude that (22) holds with $a$ and $R$.

Remark 25. If $Q$ is in $\mathcal{P}_{q}$, then instead of $Q(b x)$ we can take for $R$ the polynomial in $\mathcal{T}_{q}$ which coincides with $Q(b x)$ on the grid $\mathbb{G}_{q}$, that is, the polynomial $\boldsymbol{\Pi}_{q}(Q(b \cdot))$ of Definition [22. Indeed, it is also an idempotent polynomial since $b$ and $q$ are coprime.

So now it makes sense to formally define the following concentration coefficient. 
Definition 26. We define, for $q \in \mathbb{N}$,

$$
c_{p}^{\sharp}(q):=\sup _{R \in \mathcal{P}} \frac{\left|R\left(\frac{1}{q}\right)\right|^{p}}{\sum_{k=0}^{q-1}\left|R\left(\frac{k}{q}\right)\right|^{p}},
$$

and

$$
c_{p}^{\sharp}:=\liminf _{q \rightarrow \infty} c_{p}^{\sharp}(q) .
$$

We want to extend concentration results on discrete point grids to the whole of $\mathbb{T}$, and keep track of constants. We state this as a proposition.

Proposition 27. Let $p>0$ be such that there is full $p$-concentration with gap at 0 . If $c_{p}^{\sharp}>0$, then $p$-concentration holds for the whole of $\mathbb{T}$, and we have the inequality

$$
c_{p} \geq 2 c_{p}^{\sharp} .
$$

Moreover, the same level of concentration holds with gap.

Proof. Let us fix a symmetric open set $E$ and construct a related peaking idempotent. First, there exists some interval $J:=\left[\frac{a}{q}-\frac{1}{2 q}, \frac{a}{q}+\frac{1}{2 q}\right]$ with $(a, q)=1$, such that $J$ and $-J$ are contained in $E$. We fix $R$ that gives the $p$-concentration at $a / q$ on $\mathbb{G}_{q}$ with a constant $C$ : this can be done with $C$ arbitrarily close to $c_{p}^{\sharp}(q)$ in view of Lemma 24 .

Now, let $\varepsilon$ be given. By uniform continuity we may choose $0<\delta<$ $1 / 2$ so that we have the inequalities

$$
|R(t+a / q)|^{p} \geq|R(a / q)|^{p}-\varepsilon|R(a / q)|^{p}, \quad(|t| \leq \delta / q)
$$

and, for $k=0,1, \cdots, q-1$,

$$
|R(t+k / q)|^{p} \leq|R(k / q)|^{p}+\varepsilon|R(0)|^{p}, \quad(|t| \leq \delta / q)
$$

which implies immediately

$$
\sum_{k=0}^{q-1}|R(t+k / q)|^{p} \leq(1+q \varepsilon) \sum_{k=0}^{q-1}|R(k / q)|^{p} . \quad(|t| \leq \delta / q)
$$

Once $\delta$ is chosen, we will take $T$ a gap-peaking idempotent at 0 , provided by Proposition 9 - compare also (14)-(15) - with the given $\varepsilon, \delta$ as above, and $N$ larger than the degree of $R$, so that

$$
S(x):=R(x) T(q x)
$$

is an idempotent, too. It remains to show

$$
2 C \int_{\mathbb{T}}|S|^{p} \leq \kappa(\varepsilon) \int_{E}|S|^{p},
$$

with $\kappa(\varepsilon)$ getting arbitrarily close to 1 when $\varepsilon$ is chosen appropriately small. 
Denoting $\tau^{p}:=\int_{\mathbb{T}}|T|^{p}$ and $I:=\left[\frac{a}{q}-\frac{\delta}{2 q}, \frac{a}{q}+\frac{\delta}{2 q}\right]$, we find

$$
\begin{aligned}
\frac{1}{2} \int_{E}|S|^{p} \geq \int_{J}|S|^{p} & \geq(1-\varepsilon)|R(a / q)|^{p} \int_{I}|T(q x)|^{p} d x \\
& \geq(1-\varepsilon)|R(a / q)|^{p} \frac{1}{q} \int_{-\delta}^{\delta}|T|^{p} \\
& \geq \frac{(1-\varepsilon)^{2} \tau^{p}}{q}|R(a / q)|^{p} .
\end{aligned}
$$

We now estimate the whole integral of $|S|^{p}$. We define the intervals

$$
J_{k}:=\left[\frac{k}{q}-\frac{1}{2 q}, \frac{k}{q}+\frac{1}{2 q}\right], \quad I_{k}:=\left[\frac{k}{q}-\frac{\delta}{q}, \frac{k}{q}+\frac{\delta}{q}\right] \quad(k=0, \ldots, q-1) .
$$

Then, if we proceed as in (32), using (29) this time, we find that

$$
\sum_{k=0}^{q-1} \int_{I_{k}}|S|^{p}=\int_{I_{0}} \sum_{k=0}^{q-1}|R(t+k / q)|^{p}|T(q t)|^{p} \leq \frac{\tau^{p}}{q}(1+q \varepsilon) \sum_{k=0}^{q-1}|R(k / q)|^{p},
$$

while

$$
\begin{aligned}
\int_{J_{k} \backslash I_{k}}|S|^{p} & \leq 2|R(0)|^{p} \int_{\frac{k}{q}+\frac{\delta}{q}}^{\frac{k}{q}+\frac{1}{2 q}}|T(q x)|^{p} d x=\frac{2}{q}|R(0)|^{p} \int_{\frac{\delta}{q}}^{\frac{1}{2}}|T(x)|^{p} d x \\
& \leq \frac{\varepsilon \tau^{p}}{q}|R(0)|^{p} \leq \frac{\varepsilon \tau^{p}}{q} \sum_{k=0}^{q-1}|R(k / q)|^{p} .
\end{aligned}
$$

Taking the sum over $k$ for the last integrals and adding the above sum for integrals over the $I_{k}$ 's, we obtain the estimate

$$
\int_{\mathbb{T}}|S|^{p} \leq \frac{\tau^{p}}{q}(1+2 q \varepsilon) \sum_{k}|R(k / q)|^{p} .
$$

Combining (32) and (33), (31) obtains with $\kappa(\varepsilon):=(1-\varepsilon)^{-2}(1+2 q \varepsilon)$.

Let us finally prove $p$-concentration with gap. It is sufficient to remark that instead of taking the polynomial $R$ in (30) we could have as well taken the polynomial $R((M q+1) x)$, with $M$ arbitrarily large. From this point, the proof is identical, since the two polynomials take the same values on the grid. If the gaps of the peaking idempotent $T$ are taken large enough, then $S$ will have gaps larger than $M$.

We can modify slightly the previous proof of Proposition 27 to prove concentration results on the corresponding second grid, using the peaking property with gap at $1 / 2$ instead of 0 . 
Definition 28. We shall say that there is p-concentration at $\frac{2 a+1}{2 q}$ on the grid $\mathbb{G}_{q}^{\star}$ with constant $c$ if there exists an idempotent polynomial $R$ such that

$$
\left|R\left(\frac{2 a+1}{2 q}\right)\right|^{p}>c \sum_{k=0}^{q-1}\left|R\left(\frac{2 k+1}{2 q}\right)\right|^{p} .
$$

Remark that in particular we restrict to idempotents $R$ that do not vanish identically on the grid under consideration, which we assume in the following definition.

Definition 29. If $q \in \mathbb{N}$, then we define

$$
c_{p}^{\star}(q):=\sup _{R \in \mathcal{P}} \frac{\left|R\left(\frac{1}{2 q}\right)\right|^{p}}{\sum_{k=0}^{q-1}\left|R\left(\frac{2 k+1}{2 q}\right)\right|^{p}}
$$

and

$$
c_{p}^{\star}:=\liminf _{q \rightarrow \infty} c_{p}^{\star}(q) .
$$

Again, the first step is to restrict to $1 /(2 q)$.

Lemma 30. Assume that there is p-concentration at $1 /(2 q)$ on $\mathbb{G}_{q}^{\star}$ with constant $c$. Let now $a \in \mathbb{N}, 0 \leq a<q$ be so that $2 a+1$ and $q$ are relatively prime. Then there is also p-concentration at $(2 a+1) /(2 q)$ on the grid $\mathbb{G}_{q}^{\star}$ with the same constant $c$.

Proof. Let $Q$ be the idempotent that satisfies (34) with $a=0$. We then have a multiplicative inverse $b$ of $2 a+1 \bmod 2 q$ so that $1 \leq b<2 q$ and $(2 a+1) b \equiv 1 \bmod 2 q$; hence, in particular, also $b$ is odd. Now with this particular $b$ we can consider $R(x):=Q(b x)$ exactly as before in (24).

Clearly we have $R(0)=Q(0), R((2 a+1) /(2 q))=Q((2 a+1) b /(2 q))=$ $Q(1 /(2 q))$, and the values of $R(j /(2 q))=Q(j b /(2 q))$ with $j=0, \ldots, 2 q-$ 1 will cover all values of $Q(k /(2 q))$ with $k=0,1, \ldots, 2 q-1$, exactly once each, and such a way, that odd $j$ 's correspond to odd $k$ 's. Therefore, we conclude that (34) holds with $2 a+1$ and $R$.

Remark 31. As in Remark 25, if $Q$ is in $\mathcal{P}_{2 q}$ then instead of $Q(b x)$ we can take for $R$ the polynomial $\boldsymbol{\Pi}_{2 q}(Q(b \cdot))$, which coincides with $Q(b x)$ at each point of the grid $\mathbb{G}_{2 q}$, hence a priori on $\mathbb{G}_{q}^{\star}$.

The corresponding proposition goes as follows:

Proposition 32. Let $p>0$ be such that there is full p-concentration with gap at $1 / 2$. If $c_{p}^{\star}>0$, then $p$-concentration holds for the whole of $\mathbb{T}$ and we have the inequality

$$
c_{p} \geq 2 c_{p}^{\star}
$$

Moreover, the same property holds with arbitrarily large gaps. 
Proof. Similarly to the above, it suffices to derive the concentration phenomenon for the symmetrized of an interval $J:=\left[\frac{a}{q}, \frac{(a+1)}{q}\right]$ for $q$ a sufficiently large number, $2 a+1$ coprime to $2 q$.

In this setup for any $c<c_{p}^{\star}(q)$ Lemma 30 leads to the inequality

$$
\left|R\left(\frac{2 a+1}{2 q}\right)\right|^{p}>c \sum_{k=0}^{q-1}\left|R\left(\frac{2 k+1}{2 q}\right)\right|^{p} .
$$

with an appropriate $R \in \mathcal{P}$.

At this point, the proof is exactly the same as the one of the previous proposition, considering intervals $I_{k}$ centered at $(2 k+1) /(2 q)$ with radius $\delta / q$, with $\delta$ small enough so that $R$ is nearly constant on $I_{k}$, and then considering $S(x):=R(x) \cdot T(q x)$ again, where $T$ is now a gap-peaking idempotent at $1 / 2$, with gaps sufficiently large, so that $S$ is still an idempotent. Using the fact that outside $I_{k}$ but within $(k / q,(k+1) / q)$, the integral of $T$ is arbitrarily small in view of the peaking property at $1 / 2$, we obtain the assertion as before. The only difference is the fact that 0 is no more in the grid, so that the quotient of $R(0)^{p}$ with $\sum_{k=0}^{q-1}|R((2 k+1) /(2 q))|^{p}$ appears in the rests, but does not change the limit since it remains fixed while $\varepsilon$ tends to 0 .

The $p$-concentration with gap at the same level of concentration is obtained also in a similar way.

\section{5. p-CONCENTRATION BY MEANS OF PEAKING AT $1 / 2$}

We now prove the part of Theorem 7 concerning $p$ not an even integer, which we state separately for the reader's convenience. The following proof contains also the one of Proposition 12, which we gave in the introduction as a hint for the methods.

Proposition 33. Let $p>0$ be a given value for which there is full p-concentration with gap at $1 / 2$. Then for each nonempty symmetric open set $E \subset \mathbb{T}$ and each constant $c<1$ we can find an idempotent $S \in \mathcal{P}$ with the property that

$$
\int_{E}|S|^{p}>c \int_{\mathbb{T}}|S|^{p}
$$

Moreover, $S$ may be chosen with arbitrarily large gaps.

Proof. By Proposition [32, it is sufficient to prove that $c_{p}^{\star}=1 / 2$, that is,

$$
\liminf _{q \rightarrow \infty} \sup _{R \in \mathcal{P}} \frac{\left|R\left(\frac{1}{2 q}\right)\right|^{p}}{\sum_{k=0}^{q-1}\left|R\left(\frac{2 k+1}{2 q}\right)\right|^{p}}=\frac{1}{2} .
$$


We will restrict to a sub-family of polynomials in $\mathcal{P}$, obtained by products of Dirichlet kernels. Observe first that for $r<q$, the product

$$
D_{r}(x) \prod_{l=1}^{L-1} D_{r}\left(\left((2 q)^{l}+1\right) x\right)
$$

is also an idempotent polynomial, the modulus of which coincides with the $L$-th power of $\left|D_{r}\right|$ on the grid under consideration. So we are to prove also the last inequality in

$$
\frac{1}{c_{p}^{\star}}=\limsup _{q \rightarrow \infty} \frac{1}{2 c_{p}^{\star}(q)} \leq \inf _{L} \limsup _{q \rightarrow \infty} \min _{r<q} \frac{1}{2} \cdot \frac{\sum_{k=0}^{q-1}\left|D_{r}\left(\frac{2 k+1}{2 q}\right)\right|^{L p}}{\left|D_{r}\left(\frac{1}{2 q}\right)\right|^{L p}} \leq 1 .
$$

Let us define

$$
A(\lambda, r, q):=\left|\frac{\sin \left(\frac{\pi}{2 q}\right)}{\sin \left(\frac{r \pi}{2 q}\right)}\right|^{\lambda} \sum_{k \in \mathbb{N} ; k<q / 2}\left|\frac{\sin \left(\frac{(2 k+1) r \pi}{2 q}\right)}{\sin \left(\frac{(2 k+1) \pi}{2 q}\right)}\right|^{\lambda} .
$$

Substituting the explicit value of $D_{r}$ and using parity, the quantity appearing in the left hand side of (41) can be written as $A(L p, r, q)$ for $q$ even. When $q$ is odd, we have to subtract half of the term obtained for $k=(q-1) / 2$, which gives only a 0 contribution to the limit below. In any case, we have the inequality

$$
\left(\frac{1}{2 c_{p}^{\star}(q)} \leq\right) \frac{1}{2} \frac{\sum_{k=0}^{q-1}\left|D_{r}\left(\frac{2 k+1}{2 q}\right)\right|^{L p}}{\left|D_{r}\left(\frac{1}{2 q}\right)\right|^{L p}} \leq A(L p, r, q) .
$$

We then have the following lemma.

Lemma 34. For fixed $\lambda>1$, we have the inequality

$$
\limsup _{q \rightarrow \infty} \min _{r<q} A(\lambda, r, q) \leq \inf _{0<t<1 / 2} A(\lambda, t),
$$

where

$$
A(\lambda, t):=\frac{1}{(\sin (\pi t))^{\lambda}} \sum_{k=0}^{\infty}\left|\frac{\sin ((2 k+1) \pi t)}{2 k+1}\right|^{\lambda} .
$$

Proof. Let us fix $t \in(0,1 / 2)$, and consider the limit of $A(\lambda, 2[q t], q)$ when $q$ tends to $\infty$. It has the same limit as

$$
\left|\frac{\frac{\pi}{2}}{\sin (\pi t)}\right| \sum_{k=0}^{\lambda(q-1) / 2}\left|\frac{\sin \left(\frac{(2 k+1)[q t] \pi}{q}\right)}{q \sin \left(\frac{(2 k+1) \pi}{2 q}\right)}\right|^{\lambda} .
$$

As $q \sin \left(\frac{(2 k+1) \pi}{2 q}\right) \geq(2 k+1)$, Lebesgue's theorem for series justifies taking the limit termwise. This concludes the proof of the lemma. 
So in view of Lemma $341 /\left(2 c_{p}^{\star}(q)\right) \leq \inf _{L} \inf _{t} A(L p, t)$. If we take $t=1 / 4$, all the absolute values of the occurring sines in $A(\lambda, t)$ are equal, hence cancel out. It remains

$$
A(\lambda, 1 / 4)=\sum_{k}(2 k+1)^{-\lambda}=\left(1-2^{-\lambda}\right) \zeta(\lambda) .
$$

Now we can take $L$, or $\lambda=L p$, arbitrarily large. Therefore, the infimum in (41) is just 1 .

Note that we found that $1 / c_{p}^{\star} \leq \inf _{L} \inf _{t} A(L p, t)$ holds always.

Let us conclude this section by a remark that will be used later on for measurable sets, where we will not be able to consider large products of Dirichlet kernels for $p \leq 1$, and will have to restrict to two factors, that is, take $L=2$. Observe that each term $\left|\frac{\sin ((2 k+1) \pi t)}{(2 k+1) \sin (\pi t)}\right|$ is below 1 , so that $A(\lambda, t)$ and $\inf _{t} A(\lambda, t)$ are strictly decreasing functions of $\lambda$.

Moreover, $\inf _{0<t<1 / 2} A(2, t)$ can be computed explicitly. To compute the summation, we can use Plancherel Formula once we have recognized the Fourier coefficients (at $k$ and $-k$ ) of the function

$$
\frac{\pi}{2}\left(\chi_{[-t / 2, t / 2]}(x)-\chi_{[-t / 2, t / 2]}(x-1 / 2)\right) .
$$

It follows that

$$
A(2, t)=\frac{\pi^{2} t}{4 \sin ^{2}(\pi t)} .
$$

Substituting $x=\pi t$ and recalling (15) we find $1 / \min _{t} A(2, t)=2 c_{2} \approx$ $0.92 \ldots$, which is already much larger than $1 / 2$, and close to 1 .

\section{UNIFORM LOWER BOUNDS FOR p-CONCENTRATION}

We now prove the lower estimation in the $p \in 2 \mathbb{N}$ part of Theorem 7. We proceed as in the last section, using Proposition 27] instead of Proposition 32, since we have now gap-peaking idempotents at 0 . Similarly to the above, we consider a product of Dirichlet kernels:

$$
R(x):=D_{r}(x) \prod_{\ell=1}^{L-1} D_{r}\left(\left(q^{\ell}+1\right) x\right) .
$$

We have to consider the quantities (25) and (26), i.e. we are to calculate

$$
\frac{2}{c_{p}} \leq \limsup _{q \rightarrow \infty} \frac{1}{c_{p}^{\sharp}(q)} \leq \inf _{L} \limsup _{q \rightarrow \infty} \min _{r<q} \frac{\sum_{k=0}^{q-1}\left|D_{r}\left(\frac{k}{q}\right)\right|^{L p}}{\left|D_{r}\left(\frac{1}{q}\right)\right|^{L p}} .
$$


As before, in order to estimate the quotient in (48) we have to consider the equivalent quantity $B(L p, r, q)$ defined by

$$
B(\lambda, r, q):=\left|\frac{r \sin \left(\frac{\pi}{q}\right)}{\sin \left(\frac{r \pi}{q}\right)}\right|^{\lambda}+2\left|\frac{\sin \left(\frac{\pi}{q}\right)}{\sin \left(\frac{r \pi}{q}\right)}\right|^{\lambda} \sum_{k=1}^{q / 2}\left|\frac{\sin \left(\frac{k r \pi}{q}\right)}{\sin \left(\frac{k \pi}{q}\right)}\right|^{\lambda} .
$$

We then have the following lemma.

Lemma 35. For fixed $\lambda>1$, we have the inequality

$$
\limsup _{q \rightarrow \infty} \min _{r<q} B(\lambda, r, q) \leq \inf _{0<t<1 / 2} B(\lambda, t),
$$

where

$$
B(\lambda, t):=\left(\frac{\pi t}{\sin \pi t}\right)^{\lambda}\left(1+2 \sum_{k=1}^{\infty}\left|\frac{\sin (k \pi t)}{k \pi t}\right|^{\lambda}\right) .
$$

Proof. For fixed $t \in(0,1 / 2)$, the left hand side of (50) is bounded by the value that we obtain when letting $q \rightarrow \infty$ with $r / q$ tending to $t$ at the same time. We conclude as in Lemma 34.

Let us define for any fixed value of $\kappa>0$, the quantity

$$
\beta(\kappa):=\limsup _{\lambda \mapsto \infty} B(\lambda, \kappa \sqrt{6 / \lambda}),
$$

which will be useful later on, since $2 / c_{p} \leq \inf _{L} \inf _{t} B(L p, t) \leq \beta(\kappa)$. For fixed $s$, the quantity $(\sqrt{\lambda} / s \cdot \sin (s / \sqrt{\lambda}))^{\lambda}$ tends to $\exp \left(-s^{2} / 6\right)$. We use this for the computation of $\beta(\kappa)$ and see that the first factor of (51) tends to $\exp \left(\kappa^{2} \pi^{2}\right)$.

Applying the well-known Weierstrass product for sin we get

$$
\log \left(\frac{\sin x}{x}\right)=\sum_{n=1}^{\infty} \log \left(1-\frac{x^{2}}{n^{2} \pi^{2}}\right) \leq-\left(\sum_{n=1}^{\infty} \frac{1}{n^{2}}\right) \frac{x^{2}}{\pi^{2}}=-\frac{x^{2}}{6} .
$$

For the $\log$ function here we must restrict to $0<x<\pi$ : that provides us the useful inequality

$$
\frac{\sin x}{x} \leq \exp \left(-\frac{x^{2}}{6}\right) \quad(0<x<\pi)
$$

what we apply in the second factor of (51) for the range $1 \leq k<1 / t$. Thus (at the end extending the sum up to $\infty$ ) we are led to

$$
\sum_{k<1 / t}\left|\frac{\sin (k \pi t)}{k \pi t}\right|^{\lambda} \leq \sum_{k<1 / t} \exp \left(-\lambda \frac{k^{2} \pi^{2} t^{2}}{6}\right) \leq \sum_{k=1}^{\infty} e^{-\kappa^{2} k^{2} \pi^{2}} .
$$

Using the trivial bound $|\sin u| \leq 1$, the tail sum can be estimated as

$$
\sum_{k \geq 1 / t}\left|\frac{\sin (k \pi t)}{k \pi t}\right|^{\lambda} \leq(\pi t)^{-\lambda}\left(t^{\lambda}+\int_{1 / t}^{\infty} \frac{d u}{u^{\lambda}}\right)=\pi^{-\lambda}\left(1+\frac{1 / t}{\lambda-1}\right)
$$


which tends to 0 with $t=\kappa \sqrt{6 / \lambda}$ and $\lambda \rightarrow \infty$.

Collecting the above estimates for $\beta:=\inf _{\kappa>0} \beta(\kappa)$, we are led to

$$
\beta \leq \inf _{\kappa>0} e^{\pi^{2} \kappa^{2}}\left\{1+2 \sum_{k=1}^{\infty} e^{-\kappa^{2} k^{2} \pi^{2}}\right\} .
$$

Note that the sum in the last curly brackets is well-known as Jacobi's theta function. Choosing here $\kappa=0.225$, we can compute $\beta \leq 4.13273$, which leads to $c_{p} \geq 2 / \beta \geq 0.48394$, surprisingly close to the theoretical upper bound of $1 / 2$.

The computation of $\inf _{0<t<1 / 2} B(\lambda, t)$ can be executed explicitly for $\lambda=4$. We recognize the Fourier coefficients of the convolution product $\chi_{[-t / 2, t / 2]} * \chi_{[-t / 2, t / 2]}$, whose $L^{2}$ norm is equal to $\left(2 t^{3} / 3\right)^{1 / 2}$. Then we use the Plancherel Formula and obtain that

$$
c_{4} \geq \max _{0<t<1 / 2} \frac{3\left(\sin ^{4}(\pi t)\right)}{\pi^{4} t^{3}}>0.495,
$$

the concrete numerical value having been obtained for the choice of $t=0.267$.

Comparing the results of the last two sections, it should become clear why gap-peaking at $1 / 2$ is even more useful for us, than gap-peaking at 0 . Indeed, once we can apply gap-peaking at $1 / 2$, we are able to consider $\mathbb{G}_{q}^{\star}$ in place of $\mathbb{G}_{q}$ : and that means that instead of the second largest term $\left|D_{r}(1 / q)\right|$, we can consider the very largest term $\left|D_{r}(1 / 2 q)\right|$ in comparison to the whole grid sum. Thus in the translated grid case we can take advantage of considering arbitrarily large powers $L$, eventually killing all other terms compared to our $\left|D_{r}(1 / 2 q)\right|^{L}$, while in the original grid $\mathbb{G}_{q}$ this is subject to a fine balance, restricted by the necessity of keeping control of the dominance of the very largest term $D_{r}(0)^{L}$.

\section{Part III : Concentration for measurable sets}

We will go back to all steps of the previous proofs in order to partly generalize the results to measurable sets. We start by using the theorem of Khintchine on diophantine approximation, see [20]. We prove that a symmetric measurable set of positive measure contains large parts of intervals which are centered at a point of one of the two grids, $\mathbb{G}_{q}$ or $\mathbb{G}_{q}^{\star}$. This is done in Section 7 . Then in Section 8 we prove the gap-peaking property at 0 or $1 / 2$ in the even stronger form that some measurable set of measure $2 \eta \delta$ can be deleted from the interval $[-\delta,+\delta]$. In Section 9 we prove that values of an idempotent, concentrating on the grid, does not take too different values on the intervals of length $2 \delta$. Here we may consider additional assumptions on the degree of the polynomials. Based on the results of these sections, we will prove $p$-concentration for 
measurable sets when $p>1 / 2$, with some estimates on constants. We conclude the proof of Theorem 8 finally in $\$ 10$.

\section{The use of Diophantine Approximation}

We will state two propositions, used respectively on $\mathbb{G}_{q}$ and $\mathbb{G}_{q}^{\star}$. The first one is a direct corollary of Khintchine's Theorem, while the second one is its inhomogeneous extension, first proved by Szüsz [28] and later generalized by Schmidt [27].

Proposition 36. Let $E$ be a measurable set of positive measure in $\mathbb{T}$. For all $\theta>0, \eta>0$ and $Q \in \mathbb{N}$, there exists an irreducible fraction $k / q$ such that $q>Q$ and

$$
\left|\left[\frac{k}{q}-\frac{\theta}{q^{2}}, \frac{k}{q}+\frac{\theta}{q^{2}}\right] \cap E\right| \geq(1-\eta) \frac{2 \theta}{q^{2}} .
$$

Moreover, given a positive integer $\nu$, it is possible to choose $q$ such that $(\nu, q)=1$.

Proposition 37. Let $E$ be a measurable set of positive measure in $\mathbb{T}$. For all $\theta>0, \eta>0$ and $Q \in \mathbb{N}$, there exists an irreducible fraction $(2 k+1) /(2 q)$ such that $q>Q$ and

$$
\left|\left[\frac{2 k+1}{2 q}-\frac{\theta}{q^{2}}, \frac{2 k+1}{2 q}+\frac{\theta}{q^{2}}\right] \cap E\right| \geq(1-\eta) \frac{2 \theta}{q^{2}} .
$$

Moreover, given a positive integer $\nu$, it is possible to choose $q$ such that $(\nu, q)=1$.

Proof of Propositions [36 and 37. Let $\alpha$ be 0 or $1 / 2$. Then according to Szüsz' Theorem [28] for $\xi$ belonging to a set of full measure,

$$
\|q \xi-\alpha\| \leq \frac{\theta}{q}
$$

has an infinite number of solutions. For $\alpha=1 / 2$, for instance, it means that with a certain $k \in \mathbb{N}(0 \leq k<q)$ we have

$$
|q \xi-1 / 2-k|<\frac{\theta}{q}, \quad \text { i.e. } \quad\left|\xi-\frac{2 k+1}{2 q}\right|<\frac{\theta}{q^{2}} .
$$

We may assume, and we will do it, that the denominator and numerator are coprime: if not, we cancel out the common factors, and the error, compared to the new denominator $q^{\prime}$, is even better. Note that for irrational $\xi$ we have infinitely many different such denominators $q^{\prime}$ : indeed, if not we get a contradiction with the fact that the error tends to zero with $q$.

Let us choose for $\xi$ an irrational density point of $E$ having infinitely many solutions of (59). This we can do, since almost every point of $E$ is such. For $\eta$ fixed and $q$ sufficiently large we then have

$$
\left|\mathbb{T} \backslash E \cap\left[\xi-\frac{2 \theta}{q^{2}}, \xi+\frac{2 \theta}{q^{2}}\right]\right| \leq \frac{2 \eta \theta}{q^{2}} .
$$


So, if $q$ and $k$ are such that (60) holds and if $q$ is large enough, then (58) is satisfied by the triangle inequality.

It remains to prove that the denominators $q$ can be taken so that $(\nu, q)=1$. Schmidt proves in [27] that, for each polynomial $P$ with integer coefficients and each $\alpha \in \mathbb{T}$, for almost every $\xi$ one can find an infinite number of integers $r$ such that

$$
\|P(r) \xi-\alpha\| \leq \frac{\theta}{r}
$$

Both for $\alpha=0$ or $1 / 2$, it suffices to consider $P(r)=\nu r+1$. Schmidt's Theorem then allows (61) for a.e. $\xi$ by infinitely many $r$. So we can approach $\xi$ for $\alpha=0$ by fractions $k /(\nu r+1)$, and for $\alpha=1 / 2$ by fractions $\frac{2 k+1}{2(\nu r+1)}$, eventually simplified. So the denominator and $\nu$ will always remain coprime. The rest of the proof is identical.

\section{Peaking idempotents at 0 And $1 / 2$}

We will prove the following, which is a more accurate statement than those of Section 3 .

Proposition 38. Let $p>2$. For $\varepsilon>0$ there exists $\delta_{0}>0$ and $\eta>0$ such that, for all $\delta<\delta_{0}$ and $N \in \mathbb{N}$, if $E$ is a measurable set that satisfies $|E \cap[-\delta, \delta]|>2(1-\eta) \delta$, then there exists an idempotent $T$ with gaps larger than $N$ such that

$$
\int_{E \cap[-\delta, \delta]}|T|^{p}>(1-\varepsilon) \int_{0}^{1}|T|^{p} .
$$

Let $p>0$ not an even integer. Then for $\varepsilon>0$ there exists $\delta_{0}>0$ and $\eta>0$ such that, for all $\delta<\delta_{0}$ and $N \in \mathbb{N}$, if $E$ is a measurable set that satisfies $\left|E \cap\left[\frac{1}{2}-\delta, \frac{1}{2}+\delta\right]\right|>2(1-\eta) \delta$, then there exists an idempotent $T$ with gaps larger than $N$ such that

$$
\int_{E \cap\left[\frac{1}{2}-\delta, \frac{1}{2}+\delta\right]}|T|^{p}>(1-\varepsilon) \int_{0}^{1}|T|^{p} .
$$

Proof. We will proceed as in Section 3. The main point is, for our peaking bivariate functions $f$, to find an appropriate power $L$ of the marginal function $F$ for which the same kind of estimate is valid: we will then take a Riesz product with $L$ factors. The proposition will be a consequence of the following lemma, with $F$ the associated marginal function.

Lemma 39. Let $F:[0,1 / 2] \rightarrow[0, \infty)$ be a nonnegative, continuous function, having a strict global maximum at 0 . Moreover, assume that there exist $0<a<A$ and $\Delta>0$ with $F$ admitting the estimates

$$
F(0) \exp \left(-A x^{2}\right)<F(x)<F(0) \exp \left(-a x^{2}\right) \quad(x \in[0, \Delta]) .
$$


Then for all $\varepsilon>0$ there exists an $\eta>0$ so that for any $0<\delta<\Delta$ there is an $L=L(\varepsilon, \delta) \in \mathbb{N}$ with the property that whenever $E \subset$ $[0,1 / 2)$ is a measurable set satisfying $|E \cap[0, \delta]|>(1-\eta) \delta$, then we have the inequality

$$
\int_{E \cap[0, \delta]} F^{L}>(1-\varepsilon) \int_{0}^{1 / 2} F^{L}
$$

Remark 40. Observe that (62) certainly holds true in case F has a nonvanishing second derivative (from the right) at 0 . Also note the validity of the obvious modification for even functions on $[-1 / 2,1 / 2]$ assuming the analogous two-sided conditions.

Proof. We can assume $F(0)=1$. By condition, $\max _{[\Delta, 1 / 2]} F<1$, hence - perhaps with a different value of $a$, which still depends only on $F$ we have $F(x)<\exp \left(-a x^{2}\right)$ on the whole of $[0,1 / 2]$. Extending $F$ to the halfline $[0, \infty)$ as 0 outside $[0,1 / 2]$, we thus still have this estimate.

Let now $H:=[0, \infty) \backslash([0, \delta] \cap E)$. Then we have

$$
\int_{H} F^{L}<\int_{[0, \delta] \backslash E} 1+\int_{\delta}^{\infty} e^{-L a x^{2}} d x<|[0, \delta] \backslash E|+\int_{\delta}^{\infty} \frac{d x}{L a x^{2}}<\eta \delta+\frac{1}{\delta a L} .
$$

On the other hand with a very similar calculation we obtain

$$
\begin{aligned}
\int_{[0, \delta] \cap E} F^{L} & >\int_{[0, \delta] \cap E} e^{-L A x^{2}} d x=\left(\int_{0}^{\infty}-\int_{[0, \delta] \backslash E}-\int_{\delta}^{\infty}\right) e^{-L A x^{2}} d x \\
(64) \quad & \geq \frac{1}{2} \sqrt{\frac{\pi}{L A}}-|[0, \delta] \backslash E|-\int_{\delta}^{\infty} \frac{d x}{L a x^{2}}>\frac{1}{2} \sqrt{\frac{\pi}{L A}}-\eta \delta-\frac{1}{\delta a L} .
\end{aligned}
$$

A combination of (63) and (64) reveals that it suffices to ascertain

$$
\eta \delta<\frac{\varepsilon}{8} \sqrt{\frac{\pi}{L A}} \quad \text { and } \quad \frac{1}{\delta a L}<\frac{\varepsilon}{8} \sqrt{\frac{\pi}{L A}},
$$

that is, with a constant $C=C(a, A)=C_{F}$,

$$
\eta \delta \sqrt{L} \leq \varepsilon^{\prime} \quad \text { and } \quad \frac{1}{\delta \sqrt{L}} \leq \varepsilon^{\prime} \quad \text { with } \quad \varepsilon^{\prime}:=\varepsilon / C .
$$

Thus we conclude the proof choosing $L:=\left\lceil\varepsilon^{\prime-2} \delta^{-2}\right\rceil$ and $\eta=\varepsilon^{\prime 2} / 2$.

To prove both cases of the proposition, note that we can also translate $F$ so that the maximum point falls to $1 / 2$ instead of 0 .

At this point the proof of the proposition is identical to the proofs of Section 3, using Lemma 17. For the given $E$, we find an idempotent $T$ such that integrals of $|T|^{p}$, respectively on $E \cap[-\delta,+\delta]$ and on the 
whole torus, satisfy the same inequality as the corresponding integrals for the function $F^{L}$.

\section{Bernstein-type inequalities}

In order to adapt our proof of Proposition 27, we need to control the error done when replacing values of idempotents in a neighborhood of one of the grids by its values on the grid.

We introduce the following notation, which will simplify the proofs. For $f$ a periodic function, we will use the sums of its values on the two grids, which we denote by

$$
\Sigma_{q}(f):=\sum_{k=0}^{q-1} f\left(\frac{k}{q}\right), \quad \Sigma_{q}^{\star}(f):=\sum_{k=0}^{q-1} f\left(\frac{2 k+1}{2 q}\right) .
$$

The aim of this paragraph is to recall classical inequalities, and modify them according to our purposes. Let us prove the following lemma.

Lemma 41. For $1<p<\infty$ there exists a constant $C_{p}$ such that, for $P \in \mathcal{T}_{q}$ and for $|t|<1 / 2$, we have the two inequalities

$$
\begin{gathered}
\sum_{k=0}^{q-1}|P(t+k / q)|^{p} \leq C_{p} \sum_{k=0}^{q-1}|P(k / q)|^{p}, \\
\left.\sum_{k=0}^{q-1}|| P(t+k / q)\right|^{p}-\left.|P(k / q)|^{p}\left|\leq C_{p}\right| q t\left|\sum_{k=0}^{q-1}\right| P(k / q)\right|^{p} .
\end{gathered}
$$

Proof. For $1<p<\infty$, the $L^{p}$ norm of a trigonometric polynomial in $\mathcal{T}_{q}$ is equivalent to the $\ell^{p}$ norm of its values on the grid $\mathbb{G}_{q}$. This is known as the Marcinkiewicz-Zygmund Theorem: the implied constants depend only on $p$ but tend to $\infty$ for $p$ tending to 1 or $\infty$. For the exact form fitting to our Taylor polynomials see Theorem (7.10), p. 30 chapter $X$ in [34]; see also [25] for recent extensions. Inequality (66) then follows using the Marcinkiewicz-Zygmund Theorem twice, and invariance by translation of the $L^{p}$ norm.

To obtain (67), we use a variant of Bernstein's Inequality, which may be stated, for $P \in \mathcal{T}_{q}$, as

$$
\int_{0}^{1}|P(x+t)-P(x)|^{p} d x \leq(2 \pi q|t|)^{p} \int_{0}^{1}|P(x)|^{p} d x .
$$

Since this is not the usual form of Bernstein's Inequality, we indicate how to obtain it. We write, for positive $t$,

$$
|P(x+t)-P(x)|^{p} \leq t^{p-1} \int_{x}^{x+t}\left|P^{\prime}(u)\right|^{p} d u
$$


apply this estimate on the left hand side of $(\underline{68})$ and then change the order of integration. We then conclude by using Bernstein's Inequality as stated in Theorem (3.16), chapter $\mathrm{X}$ in [34], that is,

$$
\int_{0}^{1}\left|P^{\prime}(x)\right|^{p} d x \leq(2 \pi q)^{p} \int_{0}^{1}|P(x)|^{p} d x .
$$

Let us proceed with the proof of (67). By using the MarcinkiewiczZygmund Theorem for both sides of (68), we find that, for $1<p<\infty$, there exists some constant $C_{p}$, (independent of $P \in \mathcal{T}_{q}$ ), such that

$$
\sum_{k=0}^{q-1}|P(t+k / q)-P(k / q)|^{p} \leq C_{p}|q t|^{p} \sum_{k=0}^{q-1}|P(k / q)|^{p} .
$$

Let us use the elementary inequality

$$
\left.|| a\right|^{p}-|b|^{p}|\leq p| a-b \mid\left(|a|^{p-1}+|b|^{p-1}\right)
$$

and the Hölder Inequality together with (69), as well as our notation given in (65). We obtain the estimate

$$
\begin{aligned}
& \left.\sum_{k=0}^{q-1}|| P(t+k / q)\right|^{p}-|P(k / q)|^{p} \mid \leq \\
& \quad p C_{p}^{\frac{1}{p}}|q t|\left(\Sigma_{q}\left(|P|^{p}\right)\right)^{\frac{1}{p}} \cdot\left(\boldsymbol{\Sigma}_{q}\left(\left(|P|^{p-1}+|P(t+\cdot)|^{p-1}\right)^{\frac{p}{p-1}}\right)\right)^{\frac{p-1}{p}} .
\end{aligned}
$$

After having used Minkowski's inequality and the estimate (66), i.e. $\boldsymbol{\Sigma}_{q}\left(|P(t+\cdot)|^{p}\right) \leq C_{p} \boldsymbol{\Sigma}_{q}\left(|P|^{p}\right)$, the last factor on the right hand side becomes $C_{p}^{\prime}\left(\Sigma_{q}\left(|P|^{p}\right)\right)^{\frac{p-1}{p}}$, which concludes the proof of ([67).

The following is an easy consequence of Lemma 41.

Lemma 42. For $1<p<\infty$ and with the same constant $C_{p}$ as in Lemma 41 we have the following property. Whenever $P \in \mathcal{T}_{2 q}$ satisfies

$$
\sum_{k=0}^{q-1}|P(k / q)|^{p} \leq K \Sigma_{q}^{\star}\left(|P|^{p}\right),
$$

then, for any $|t|<1 / 2$, we have the two inequalities

$$
\begin{gathered}
\sum_{k=0}^{2 q-1}\left|P\left(\frac{k}{2 q}+t\right)\right|^{p} \leq C_{p}(K+1) \Sigma_{q}^{\star}\left(|P|^{p}\right), \\
\left.\sum_{k=0}^{2 q-1}|| P\left(\frac{k}{2 q}+t\right)\right|^{p}-\left|P\left(\frac{k}{2 q}\right)\right|^{p}\left|\leq 2 C_{p}(K+1)\right| q t \mid \Sigma_{q}^{\star}\left(|P|^{p}\right) .
\end{gathered}
$$

This lemma explains why we introduce the next definition. 
Definition 43. Let $0<p<\infty$ and $q \in \mathbb{N}$. We say that a polynomial $f$ satisfies the grid-condition with constant $K$, if we have

$$
\sum_{k=0}^{q-1}\left|f\left(\frac{k}{q}\right)\right|^{p} \leq K \sum_{k=0}^{q-1}\left|f\left(\frac{2 k+1}{2 q}\right)\right|^{p},
$$

that is, with the notation (65), $\Sigma_{q}\left(|f|^{p}\right) \leq K \Sigma_{q}^{\star}\left(|f|^{p}\right)$.

Remark 44. When $P \in \mathcal{T}_{q}$, (71) - i.e., the grid condition (74) for $P$ - holds with $K=C_{p}$ depending only on $p>1$ : just use (66) for the translated by $1 / 2 q$ polynomial.

We will use these considerations for products of such polynomials as well.

Lemma 45. For $1 / 2<p<\infty$ there exists a constant $A_{p}$ such that, whenever $Q \in \mathcal{T}_{2 q}$ satisfies the grid-condition (174) with exponent $2 p$, i.e.

$$
\Sigma_{q}\left(|Q|^{2 p}\right):=\sum_{k=0}^{q-1}|Q(k / q)|^{2 p} \leq K \Sigma_{q}^{\star}\left(|Q|^{2 p}\right),
$$

then for the product polynomial $R(x):=Q(x) Q((2 q+1) x)$ we have for all $|t|<1 / 2$ and for all $a:=0,1, \ldots, q-1$ the two inequalities

$$
\sum_{k=0}^{q-1}\left|R\left(t+\frac{2 k+1}{2 q}\right)\right|^{p} \leq\left(1+A_{p}(K+1) q^{2}|t|\right) \Sigma_{q}^{\star}\left(|Q|^{2 p}\right)
$$

$$
\left|R\left(t+\frac{2 a+1}{2 q}\right)\right|^{p} \geq\left|R\left(\frac{2 a+1}{2 q}\right)\right|^{p}-A_{p}(K+1) q^{2}|t| \Sigma_{q}^{\star}\left(|Q|^{2 p}\right) .
$$

Proof. Let us put, for $k=0,1, \ldots 2 q-1$,

$$
X_{k}(t):=\left|Q\left(t+\frac{k}{2 q}\right)\right|^{2 p} .
$$

Note that the two factors of $R$ take the same values on the grid $\mathbb{G}_{q}^{\star}$. Moreover, since $Q \in \mathcal{T}_{2 q}$ and $2 p>1$, it follows from Lemma 42 , formula (73) that

$$
\sum_{k=0}^{2 q-1}\left|X_{k}(t)-X_{k}(0)\right| \leq 2 C_{2 p}(K+1) q|t| \Sigma_{q}^{\star}\left(|Q|^{2 p}\right) .
$$

Let us pass to

$$
Y_{k}(t):=\left|R\left(t+\frac{k}{2 q}\right)\right|^{p}=\sqrt{X_{k}(t) X_{k}((2 q+1) t)} .
$$


Using the Cauchy-Schwarz Inequality and the previous inequality, we find for all $|t| \leq 1 / 2$

$$
\begin{aligned}
\sum_{k=0}^{q-1} Y_{2 k+1}(t) & \leq\left(\sum_{k=0}^{q-1} X_{2 k+1}(t)\right)^{\frac{1}{2}}\left(\sum_{k=0}^{q-1} X_{2 k+1}((2 q+1) t)\right)^{\frac{1}{2}} \\
& \leq\left(\Sigma_{q}^{\star}\left(|Q|^{2 p}\right)+\sum_{k=0}^{2 q-1}\left|X_{k}(t)-X_{k}(0)\right|\right)^{\frac{1}{2}} \\
& \times\left(\Sigma_{q}^{\star}\left(|Q|^{2 p}\right)+\sum_{k=0}^{2 q-1}\left|X_{k}((2 q+1) t)-X_{k}(0)\right|\right)^{\frac{1}{2}} \\
& \leq\left(1+2 C_{2 p}(2 q+1) q(K+1)|t|\right) \Sigma_{q}^{\star}\left(|Q|^{2 p}\right) .
\end{aligned}
$$

We have proved (76). We can write in the same way that

$$
\begin{aligned}
Y_{2 a+1}(t)^{2} \geq\left(X_{2 a+1}(0)-\sum_{k=0}^{2 q-1}\left|X_{k}(t)-X_{k}(0)\right|\right) \\
\quad \times\left(X_{2 a+1}(0)-\sum_{k=0}^{2 q-1}\left|X_{k}((2 q+1) t)-X_{k}(0)\right|\right),
\end{aligned}
$$

so that

$$
Y_{2 a+1}(t) \geq Y_{2 a+1}(0)-2 C_{2 p}(K+1)(2 q+1) q|t| \Sigma_{q}^{\star}\left(|Q|^{2 p}\right) .
$$

10. FRom DisCRETE CONCENTRATION TO CONCENTRATION FOR MEASURABLE SETS

Definition 46. We define

$$
\gamma_{p}^{\sharp}:=\liminf _{q \rightarrow \infty} \gamma_{p}^{\sharp}(q), \quad \gamma_{p}^{\sharp}(q):=\sup _{R \in \mathcal{P}_{q}} \frac{\left|R\left(\frac{1}{q}\right)\right|^{p}}{\sum_{k=0}^{q-1}\left|R\left(\frac{k}{q}\right)\right|^{p}} .
$$

Using the notation (51), the results of Section 6 give immediately

$$
\left(\gamma_{p}^{\sharp}\right)^{-1} \leq \inf _{0<t<1 / 2} B(p, t),
$$

valid for any $p>1$.

Let us give the corresponding definition for the grid $\mathbb{G}_{q}^{\star}$.

Definition 47. We define

$$
\gamma_{p}^{\star}:=\liminf _{q \rightarrow \infty} \gamma_{p}^{\star}(q), \quad \gamma_{p}^{\star}(q):=\sup _{R \in \mathcal{P}_{q}} \frac{\left|R\left(\frac{1}{2 q}\right)\right|^{p}}{\sum_{k=0}^{q-1}\left|R\left(\frac{2 k+1}{2 q}\right)\right|^{p}} .
$$


We have seen in Section 5 that, for $p>1$, with the notation (45),

$$
\left(\gamma_{p}^{\star}\right)^{-1} \leq \inf _{0<t<1 / 2} A(p, t)
$$

Proposition 48. Let $p>1 / 2$ not an even integer. Then there is $p$ concentration for measurable sets, and $\gamma_{p} \geq 2 \gamma_{2 p}^{\star}$; furthermore, there is $p$ concentration for measurable sets with gap at the same level.

Proof. The proof is organized as the one of Proposition 27. At the outset we have a measurable and symmetric set $E \subset \mathbb{T}$ with $|E|>$ 0 . Let us first take $C<\gamma_{2 p}^{\star}$ arbitrarily close to $\gamma_{2 p}^{\star}$, then fix $\varepsilon$ a small constant. Let $\eta$ and $\delta_{0}$ be given by Proposition 38 (second case), depending on $\varepsilon$. Let $\theta>0$ be a small constant which will be fixed later on, and $q_{0}$ large enough so that, for $q>q_{0}$ one has $C<\gamma_{2 p}^{\star}(q)$ and $\theta / q \leq \delta_{0}$. With this data we consider some interval centered at $(2 a+1) /(2 q)$ given by Proposition 37 . Let $P \in \mathcal{P}_{q}$ be such that

$$
\left|P\left(\frac{1}{2 q}\right)\right|^{2 p}>C \sum_{k=0}^{q-1}\left|P\left(\frac{2 k+1}{2 q}\right)\right|^{2 p}=C \Sigma_{q}^{\star}\left(|P|^{2 p}\right) .
$$

By Lemma [30, and Remark 31, we can find an idempotent $Q \in \mathcal{P}_{2 q}$ such that we have

$$
\left|Q\left(\frac{2 a+1}{2 q}\right)\right|^{2 p}=\left|P\left(\frac{1}{2 q}\right)\right|^{2 p}
$$

and

$$
\sum_{k=0}^{q-1}\left|Q\left(\frac{2 k+1}{2 q}\right)\right|^{2 p}=\sum_{k=0}^{q-1}\left|P\left(\frac{2 k+1}{2 q}\right)\right|^{2 p}
$$

and also

$$
\sum_{k=0}^{q-1}\left|Q\left(\frac{k}{q}\right)\right|^{2 p}=\sum_{k=0}^{q-1}\left|P\left(\frac{k}{q}\right)\right|^{2 p} .
$$

Recall that $P \in \mathcal{P}_{q}$, so for $2 p>1$ according to Remark 44 it satisfies the grid condition (74) with a constant $C_{2 p}$ depending only on $p$. Since $P$ and $Q$ attain exactly the same set of values both on the two grids $\mathbb{G}_{q}$ and $\mathbb{G}_{q}^{\star}$, the idempotent $Q$ also satisfies the grid-condition (174) for $2 p$ with the constant $C_{2 p}$. So the idempotent

$$
R(x):=Q(x) Q((2 q+1) x),
$$

matching with $Q^{2}$ on both grids, also satisfies

$$
|R(0)|^{p} \leq \boldsymbol{\Sigma}_{q}\left(|R|^{p}\right) \leq C_{2 p} \Sigma_{q}^{\star}\left(|R|^{p}\right),
$$

i.e. the grid condition (74) holds for $R$, too (with $K=C_{2 p}$ ). Whence Lemma 45 applies to $R$, so choosing $\theta$ satisfying $A_{p}\left(C_{2 p}+1\right) C^{-1} \theta \leq \varepsilon$ 
and in view of (84), (85) and (86) for all $|t|<\theta / q^{2}$ we obtain the estimates

$$
\begin{gathered}
\sum_{k=0}^{q-1}\left|R\left(t+\frac{2 k+1}{2 q}\right)\right|^{p} \leq(1+\varepsilon) \Sigma_{q}^{\star}\left(|P|^{2 p}\right)=(1+\varepsilon) \Sigma_{q}^{\star}\left(|R|^{p}\right), \\
\left|R\left(t+\frac{2 a+1}{2 q}\right)\right|^{p} \geq(1-\varepsilon)\left|R\left(\frac{2 a+1}{2 q}\right)\right|^{p}
\end{gathered}
$$

using also that, on comparing (83), (84), (85) and (87) we are led to

$$
C \Sigma_{q}^{\star}\left(|R|^{p}\right) \leq\left|R\left(\frac{2 a+1}{2 q}\right)\right|^{p} .
$$

Next, we will need a peaking idempotent at $1 / 2$, as obtained by Proposition 38. This one will depend on our given constants $\varepsilon, \eta$, $\delta=\theta / q$ and $N$ larger than the degree of $R$, and also on a measurable set of finite measure $E_{\varepsilon}$ that we define now. The mapping $x \mapsto q x$ is bijective from $J:=(k / q,(k+1) / q)$ onto $(0,1)$, and we take for $E_{\varepsilon}$ the image of $E \cap J$. It is clear that the condition

$$
\left|E_{\varepsilon} \cap\left[\frac{1}{2}-\delta, \frac{1}{2}+\delta\right]\right|>2(1-\eta) \delta
$$

has been satisfied. We take the idempotent $T$ provided by Proposition 38 for this data, satisfying

$$
\int_{E_{\varepsilon} \cap\left[\frac{1}{2}-\delta, \frac{1}{2}+\delta\right]}|T|^{p}>(1-\varepsilon) \int_{0}^{1}|T|^{p} .
$$

We finally consider the product

$$
S(x):=T(q x) R(x),
$$

which is also an idempotent. We will prove as in Section 4 that

$$
2 C \int_{\mathbb{T}}|S|^{p} \leq \kappa(\varepsilon) \int_{E}|S|^{p},
$$

with $\kappa(\varepsilon)$ being arbitrarily close to 1 when $\varepsilon$ is sufficiently small. In order to do this, we put

$$
J_{k}:=\left[\frac{k}{q}, \frac{k+1}{q}\right], \quad I_{k}:=\left[\frac{2 k+1}{2 q}-\frac{\theta}{q^{2}}, \frac{2 k+1}{2 q}+\frac{\theta}{q^{2}}\right]
$$

for $k=0, \ldots, q-1$. From now on the proof of the proposition is similar to the one of Proposition 27. We repeat briefly the steps for 
the reader's convenience. Denoting $\tau^{p}:=\int_{\mathbb{T}}|T|^{p}$, we find, using the property (92), that

$$
\begin{aligned}
\frac{1}{2} \int_{E}|S|^{p} \geq \int_{I_{a} \cap E}|S|^{p} & \geq(1-\varepsilon)\left|R\left(\frac{2 a+1}{2 q}\right)\right|^{p} \int_{I_{a} \cap E}|T(q x)|^{p} d x \\
& \geq(1-\varepsilon)\left|R\left(\frac{2 a+1}{2 q}\right)\right|^{p} \frac{1}{q} \int_{E_{\varepsilon} \cap\left[\frac{1}{2}-\delta, \frac{1}{2}+\delta\right]}|T|^{p} \\
& \geq \frac{(1-\varepsilon)^{2} \tau^{p}}{q}\left|R\left(\frac{2 a+1}{2 q}\right)\right|^{p} .
\end{aligned}
$$

Then we give an upper bound for the integral on the whole torus:

$$
\begin{aligned}
\sum_{k=0}^{q-1} \int_{I_{k}}|S|^{p} & =\int_{-\theta / q^{2}}^{\theta / q^{2}} \sum_{k=0}^{q-1}\left|R\left(\frac{2 k+1}{2 q}+t\right)\right|^{p}|T(q t)|^{p} d t \\
& \leq(1+\varepsilon) \Sigma_{q}^{\star}\left(|R|^{p}\right) \frac{\tau^{p}}{q}
\end{aligned}
$$

while

$$
\begin{aligned}
\int_{J_{k} \backslash I_{k}}|S|^{p} & \leq 2\|R\|_{\infty}^{p} \int_{\frac{k}{q}+\frac{\delta}{q}}^{\frac{k}{q}+\frac{1}{2 q}}|T(q x)|^{p} d x=2|R(0)|^{p} \frac{1}{q} \int_{\frac{\delta}{q}}^{\frac{1}{2}}|T(x)|^{p} d x \\
& \leq \frac{\varepsilon \tau^{p}}{q}|R(0)|^{p} \leq \frac{C_{2 p} \varepsilon \tau^{p}}{q} \Sigma_{q}^{\star}\left(|R|^{p}\right),
\end{aligned}
$$

making use of (88), too. Summing the last integrals over $k$, we obtain

$$
\int_{\mathbb{T}}|S|^{p} \leq \frac{\tau^{p}}{q}\left(1+\varepsilon+C_{2 p} \varepsilon\right) \Sigma_{q}^{\star}\left(|R|^{p}\right) .
$$

Now (91), (96) and (97) give (94) with $\kappa(\varepsilon):=\left(1+\varepsilon+C_{2 p} \varepsilon\right)(1-\varepsilon)^{-2}$, concluding the proof, except for assuring arbitrarily large gaps.

It remains to indicate how to modify the proof to get peaking idempotents with arbitrarily large gaps. So we fix $\nu$ as a large odd integer, and we will prove that we can replace the polynomial $Q(x)$ by some polynomial $\tilde{Q}(\nu x)$, with gaps at least $\nu$. Recall first that we can take arbitrarily large $q$ satisfying $(\nu, q)=1$. So we now choose $\tilde{Q}$ similarly as before, to be the polynomial of degree $2 q$ that coincides with $P(b x)$ on the grid $\mathbb{G}_{q}$, but now with $b$ chosen so that $\nu b(2 a+1) \equiv 1 \bmod 2 q$. Such a $b$ exists, as $\nu(2 a+1)$ and $2 q$ are coprime. We then fix

$$
R(x):=\tilde{Q}(\nu x) \tilde{Q}((2 q+1) \nu x) .
$$

There is an additional factor $\nu$, which modifies the value of $\theta$, but otherwise the proof is identical. We know that $\tilde{Q}(\nu x)$ and $P(b x)$, and 
thus $P(x)$, take globally the same values on both grids $\mathbb{G}_{q}$ and $\mathbb{G}_{q}^{\star}$, because in each case we multiply by an odd integer that is coprime with $2 q$. So in particular the grid condition (75) is satisfied with $C_{2 p}$ once again.

Similarly, but with the grid $\mathbb{G}_{q}$ instead of $\mathbb{G}_{q}^{\star}$, we obtain the following.

Proposition 49. Let $p>2$ an even integer. Then there is $p$-concentration for measurable sets, and $\gamma_{p} \geq 2 \max \left(\gamma_{p}^{\sharp}, \gamma_{2 p}^{\sharp}\right)$. Moreover, we can choose the concentrating trigonometric polynomials with arbitrarily large gaps.

Proof. We do not give the proof, since most modifications are straightforward, and even simpler. Now if $\gamma_{p}^{\sharp} \geq \gamma_{2 p}^{\sharp}$, we consider $C<\gamma_{p}^{\sharp}$ and $P$ satisfying

$$
\left|P\left(\frac{1}{q}\right)\right|^{p}>C \sum_{k=0}^{q-1}\left|P\left(\frac{k}{q}\right)\right|^{p} .
$$

We build $R:=Q:=\Pi_{q} P(b \cdot)$ of degree lower than $q$, using Lemma 24 and Remark 25, with $b$ chosen such that $b \cdot a \equiv 1 \bmod q$, and thus $a / q$ is mapped on $1 / q$. Thus we obtain the required concentration as above.

If $\gamma_{p}^{\sharp}<\gamma_{2 p}^{\sharp}$, we take $C<\gamma_{2 p}^{\sharp}$ and an idempotent $P \in \mathcal{P}_{q}$ satisfying

$$
\left|P\left(\frac{1}{q}\right)\right|^{2 p}>C \sum_{k=0}^{q-1}\left|P\left(\frac{k}{q}\right)\right|^{2 p} .
$$

In this case we consider $R:=R(x):=Q(x) Q((q+1) x)$ with $Q:=$ $\Pi_{q} P(b \cdot) \in \mathcal{P}_{q}$, and the proof is even more like the above argument.

\section{Positive Definite trigonometric polynomials}

The proof of Proposition 48 generalizes directly to the class $\mathcal{P}^{+}$, with the main difference that, when considering the values of a polynomial $P$ on some grid $\mathbb{G}_{q}$ or $\mathbb{G}_{q}^{\star}$, we can always consider the projected polynomial $\boldsymbol{\Pi}_{2 q}(P)$, taking the same values on $\mathbb{G}_{2 q}$ and hence both on $\mathbb{G}_{q}$ and on $\mathbb{G}_{q}^{\star}$ : here we need not be concerned for occasional coincidences of projected terms in the sum, as the projection $\boldsymbol{\Pi}_{2 q}$ leaves $\mathcal{P}^{+}$invariant anyway. Therefore, the concentration constants $\gamma_{p}^{+}$, that we will obtain for positive definite functions and measurable sets, will be the same as the ones for open sets (i.e. $c_{p}$ ). In particular, we have the following.

Theorem 50. Let $p>0$ not an even integer. Then there is full $p$-concentration for the class $\mathcal{P}^{+}$for measurable sets. Moreover, we can choose the concentrating positive definite trigonometric polynomials with arbitrarily large gaps. 
Proof. The proof follows the same lines as the one of Proposition 48, but is simpler. We know that for $p \notin 2 \mathbb{N}$ there is full $p$-concentration at $1 / 2$, and also from Section 5 that this implies $c_{p}^{\star}=1 / 2$, c.f. the proof of Proposition 33. So it is sufficient to prove the following lemma, which is very similar to Proposition 48 .

Lemma 51. Let $p>0$. Then there is $p$-concentration for the class $\mathcal{P}^{+}$for measurable sets, and if $p \notin 2 \mathbb{N}$, then the level of concentration satisfies $\gamma_{p}^{+} \geq 2 c_{L p}^{\star}$ for any $L$ such that $L p>1$. Moreover, unless $p=2$, we can choose the concentrating trigonometric polynomials with arbitrarily large gaps.

Proof. We only sketch the modifications to accomplish in the proof of Proposition 48. Now $C<c_{L p}^{\star}$. Naturally, we choose $P \in \mathcal{P}_{q}$ such that,

$$
\left|P\left(\frac{1}{2 q}\right)\right|^{L p}>C \sum_{k=0}^{q-1}\left|P\left(\frac{2 k+1}{2 q}\right)\right|^{L p} .
$$

Then, as before, we choose $Q:=\Pi_{2 q}(P(b \cdot))$. Now we can take $R:=$ $Q^{L}$, as clearly $R \in \mathcal{P}^{+}$, and its degree is less than $2 L q$ (instead of $2 q(2 q+1)$ previously). So the Bernstein type inequalities can be applied more easily, with better estimates than previously, not restricting the value of $L$ in this case. (In fact, we could as well consider $\Pi_{2 q} R \in$ $\mathcal{T}_{2 q} \cap \mathcal{P}^{+}$, too.)

Note that here there is no need to $L \rightarrow \infty$, but only to take some $L>1 / p$, as we already have $c_{p}^{\star}=1 / 2$ for $p \notin 2 \mathbb{N}$. On the other hand $L>1 / p$ we really do need, as we apply Marcinkievicz-Zygmund inequalities in the proof.

Otherwise the proof for $L p>1$ can be adapted from Proposition 48, with all other modifications being straightforward.

When $p \in 2 \mathbb{N}$, we do not have gap-peaking at $1 / 2$, but, unless $p=2$, we have that at 0 . With a completely analogous argument, we obtain the corresponding result as follows.

Theorem 52. Let $p \neq 2$ be an even integer. Then there is $p$-concentration for the class $\mathcal{P}^{+}$for measurable sets at the level $\gamma_{p}^{+} \geq 2 \sup _{L \in \mathbb{N}} c_{L p}^{\sharp}$. Moreover, we can choose the concentrating positive definite trigonometric polynomials with arbitrarily large gaps.

\section{Concentration of RANDOM idempotents}

We will see that part of the estimates proved for $\mathcal{P}^{+}$in Section 11 extend to $\mathcal{P}$. This will be shown by certain random constructions of idempotents.

We have seen in Section [6 that $\inf _{t} B(\lambda, t)$ appears naturally when proving lower bounds for $c_{p}$ when $p>2$ is an even integer: for $c_{p}$ (and thus for $\gamma_{p}^{+}$) we obtained the lower bound $\sup _{L} 2 / \inf _{t} B(L p, t)$. We will now prove the same lower bound for $\gamma_{p}$. 
Proposition 53. Let $p>2$ an even integer. Then, for $L \geq 1$ an integer, $\gamma_{p} \geq 2 / \inf _{t} B(L p, t)$.

Proof. Let $C<1 / \min _{t} B(L p, t)=1 / B\left(L p, t_{0}\right)$, say, and let us chose some $c:=c(L, p)<t_{0}$. Then let $q$ be large enough, and $P \in \mathcal{P}_{q}$ such that

$$
\left|P\left(\frac{1}{q}\right)\right|^{L p}>C \sum_{k=0}^{q-1}\left|P\left(\frac{k}{q}\right)\right|^{L p} .
$$

Reflecting back to Section 6, we know that $P$ may be taken as some Dirichlet kernel $D_{r}$, with $r=\left[t_{0} q\right]>c q$. (This is the only specific property of $D_{r}$ that we will use.) Let us take $R:=M^{-1} \boldsymbol{\Pi}_{q}\left(P^{L}\right)$, which coincides with $M^{-1} P^{L}$ on the grid $\mathbb{G}_{q}$. Choosing $M:=L r^{L-1}$, which is a majorant of the Fourier coefficients of $\Pi_{q}\left(P^{L}\right)$, the polynomial $R$ may be written as

$$
R=\sum_{k=0}^{q-1} \alpha_{k} e_{k}
$$

with all $\alpha_{k} \in[0,1]$ and $\sum_{k} \alpha_{k}=R(0)=r / L$. By construction, we also have

$$
\left|R\left(\frac{1}{q}\right)\right|^{p}>C \sum_{k=0}^{q-1}\left|R\left(\frac{k}{q}\right)\right|^{p} .
$$

We now define a random idempotent $R_{\omega}$ by

$$
R_{\omega}=\sum_{k=0}^{q-1} X_{k}(\omega) e_{k}
$$

where $X_{k}$ are independent Bernoulli random variables, with $X_{k}$ of parameter $\alpha_{k}$, that is, $\mathbb{P}\left(X_{k}=1\right)=\alpha_{k}$. We want to prove that for any $\varepsilon>0$ and for $q>q_{0}(\varepsilon)$, with positive probability the random idempotent $R_{\omega}$ satisfies the inequality

$$
\left|R_{\omega}\left(\frac{1}{q}\right)\right|^{p}>K(\varepsilon) \sum_{k=0}^{q-1}\left|R_{\omega}\left(\frac{k}{q}\right)\right|^{p},
$$

with $K(\varepsilon):=K_{p}(\varepsilon)$ arbitrarily close to $C$ with $\varepsilon$ sufficiently small.

Observe that our random idempotents $R_{\omega}$ are such that $\mathbb{E}\left(R_{\omega}(x)\right)=$ $R(x)$, so in view of (102), in order to prove (103) we have to measure the error done when replacing $R_{\omega}$ by its expectation. Let us center our Bernoulli variables $X_{k}$ by considering $\widetilde{X}_{k}:=X_{k}-\alpha_{k}$. Clearly, $\widetilde{X}_{k}$ has variance $\mathbb{V}\left(\widetilde{X}_{k}\right)=\alpha_{k}\left(1-\alpha_{k}\right) \leq \alpha_{k}$, so $R_{\omega}(k / q)$ has expectation $R(k / q)$ and variance bounded by $r / L$. Also, by assumption, $|R(1 / q)|>$ $C^{1 / p} R(0)>\frac{c C^{1 / p}}{L} q$, so after an application of Markov's Inequality we find

$$
\mathbb{P}\left(\left|\frac{R_{\omega}(1 / q)}{R(1 / q)}\right| \leq 1-\varepsilon\right) \leq A \varepsilon^{-2} q^{-1},
$$


where $A$ depends on $p, c, L$, but is independent of $q$ and $\varepsilon$. Whence for $q$ large enough, the inequality

$$
\left|\frac{R_{\omega}(1 / q)}{R(1 / q)}\right|>1-\varepsilon
$$

holds with probability say at least $2 / 3$.

Let us now consider the sums

$$
S(\omega):=\sum_{k=0}^{q-1}\left|R_{\omega}\left(\frac{k}{q}\right)\right|^{p} \quad S:=\sum_{k=0}^{q-1}\left|R\left(\frac{k}{q}\right)\right|^{p},
$$

which we want to compare. So we also put

$$
\widetilde{R}_{\omega}(k / q):=R_{\omega}(k / q)-R(k / q), \quad \widetilde{S}(\omega):=\sum_{k=0}^{q-1}\left|\widetilde{R}_{\omega}(k / q)\right|^{p} .
$$

We claim that

$$
\mathbb{E}(\widetilde{S}(\omega)) \leq q C_{p}\left(1+\sum \alpha_{k}\right)^{\frac{p}{2}}=q C_{p}\left(1+\frac{r}{L}\right)^{\frac{p}{2}} .
$$

Let us first assume this inequality and conclude the proof of the proposition. So, using (105),$S \geq R(0)^{p}=r / L$ and $\widetilde{S}(\omega) \geq 0$ we are led to

$$
\mathbb{P}(C(\varepsilon) \widetilde{S}(\omega) \geq \varepsilon S) \leq \frac{C(\varepsilon)}{\varepsilon S} \cdot q C_{p}\left(1+\frac{r}{L}\right)^{\frac{p}{2}} \leq A \varepsilon^{-1} q^{1-p / 2} .
$$

Therefore the inequality

$$
C(\varepsilon) \widetilde{S}(\omega)<\varepsilon S
$$

also holds with probability at least $2 / 3$ for $q$ large enough.

Next we will need the elementary inequality

$$
|a|^{p} \leq(1+\varepsilon)|b|^{p}+C(\varepsilon)|a-b|^{p},
$$

valid for arbitrary $\varepsilon>0$ with some corresponding constant $C(\varepsilon)$. This is indeed obvious in case we have $|a| \leq \mu|b|$ with $\mu:=(1+\varepsilon)^{1 / p}>1$, while otherwise we can write $|a-b| \geq|a|-|b| \geq|a|(1-1 / \mu))$, therefore $|a| \leq \mu /(\mu-1)|a-b|$ and we obtain the inequality again. So applying this inequality with $a=R_{\omega}(k / q)$ and $b=R(k / q)$ we can estimate $\left|R_{\omega}(k / q)\right|^{p}$ by $(1+\varepsilon)|R(k / q)|^{p}+C(\varepsilon)\left|\widetilde{R}_{\omega}(k / q)\right|^{p}$, yielding

$$
S(\omega) \leq(1+\varepsilon) S+C(\varepsilon) \widetilde{S}(\omega) .
$$

Therefore, taking into account (106), (102) and (104), we find that

$$
C S(\omega) \leq C(1+2 \varepsilon) S<(1+2 \varepsilon)|R(1 / q)|^{p} \leq \frac{1+2 \varepsilon}{(1-\varepsilon)^{p}}\left|R_{\omega}(1 / q)\right|^{p}
$$

holds with probability at least $1 / 3$ for $q>q_{0}=q_{0}(\varepsilon, p, c, L)$.

So we find that (103) does indeed hold with $K(\varepsilon):=C(1-\varepsilon)^{p} /(1+2 \varepsilon)$ and for some appropriate idempotent $R_{\omega}$, once we have (105), which 
we prove now. This is a consequence of the following lemma, which is certainly classical, but which we give here for the reader's convenience.

Lemma 54. For $p>1$ there exists some constant $C_{p}$ with the following property. Let $\alpha_{k} \in[0,1]$ and $a_{k} \in \mathbb{C}$ be arbitrary for $k=0,1, \ldots, N$. Let $X_{k}$ be a sequence of independent Bernoulli random variables with parameter $\alpha_{k}$, and let $\widetilde{X}_{k}:=X_{k}-\alpha_{k}$ be their centered version, again for $k=0,1, \ldots, N$. Then we have

$$
\mathbb{E}\left(\left|\sum_{k=0}^{N} a_{k} \widetilde{X}_{k}\right|^{2 p}\right) \leq C_{p} \cdot \max _{k=1, \ldots, N}\left|a_{k}\right|^{2 p} \cdot\left(1+\sum_{k=0}^{N} \alpha_{k}\right)^{p} .
$$

Proof. We can normalize by taking $\max _{k=1, \ldots, N}\left|a_{k}\right|=1$. It follows from classical martingale inequalities (see [11]) that

$$
\mathbb{E}\left(\left|\sum_{k=0}^{N} a_{k} \widetilde{X}_{k}\right|^{2 p}\right) \leq A_{p} \mathbb{E}\left(\left|\sum_{k=0}^{N} \tilde{X}_{k}^{2}\right|^{p}\right) .
$$

So we are left with proving the inequality

$$
\mathbb{E}\left(\left|\sum_{k=0}^{N} \tilde{X}_{k}^{2}\right|^{p}\right) \leq A_{p}^{\prime}\left(1+\sum_{k=0}^{N} \alpha_{k}\right)^{p}
$$

If $0 \leq \alpha \leq 1$ and $Y$ is a centered Bernoulli variable with parameter $\alpha$, then

$\mathbb{E}\left(e^{Y^{2}}\right)=\alpha e^{(1-\alpha)^{2}}+(1-\alpha) e^{\alpha^{2}} \leq \alpha\left(1+e(1-\alpha)^{2}\right)+(1-\alpha)\left(1+e \alpha^{2}\right) \leq e^{e \alpha}$, because $e^{x} \leq 1+e x$ for $0 \leq x \leq 1$ and $1+e \alpha(1-\alpha) \leq 1+e \alpha \leq e^{e \alpha}$. So

$$
\mathbb{E}\left(e^{\sum_{k=0}^{N} \widetilde{X}_{k}^{2}}\right) \leq e^{e \sum_{k=0}^{N} \alpha_{k}} .
$$

Finally, we use the fact that, whenever $Z$ is a nonnegative random variable such that $\mathbb{E}\left(e^{Z}\right) \leq e^{\kappa}$, then

$$
\begin{aligned}
\mathbb{E}\left(Z^{p}\right) & =p \int_{0}^{\infty} \mathbb{P}(Z>\lambda) \lambda^{p-1} d \lambda \leq(2 \kappa)^{p}+p \int_{2 \kappa}^{\infty} e^{\kappa-\lambda} \lambda^{p-1} d \lambda \\
& \leq 2^{p} \kappa^{p}+p \int_{0}^{\infty} e^{-\lambda / 2} \lambda^{p-1} d \lambda=2^{p} \kappa^{p}+A_{p}^{\prime \prime} \leq\left(2^{p}+A_{p}^{\prime \prime}\right)(1+\kappa)^{p} .
\end{aligned}
$$

Putting $Z:=\sum_{k} \widetilde{X}_{k}^{2}$ and $\kappa:=e \sum_{k} \alpha_{k}$, (109) leads to (108).

So there exists $R_{\omega} \in \mathcal{P}_{q}$ with (103), whence $\liminf _{q \rightarrow \infty} \gamma_{p}^{\sharp}(q) \geq C$, even $\gamma^{\sharp}:=\liminf _{q \rightarrow \infty} \gamma_{p}^{\sharp}(q) \geq 1 / \inf _{t} B(L p, t)$, and referring to Proposition 49 concludes the proof of Proposition 53 . 
Note that the result implies $\gamma_{4} \geq 2 / \inf _{t} B(4, t)=0.495 \ldots$, as computed in (56) at the end of Section 6 for the sake of $c_{4}$, and similarly $\gamma_{2 k} \geq 0.483 \ldots$ for general $k>2$ according to the calculations of (55).

Remark 55. These results could also have been obtained by applying the direct estimates of Salem and Zygmund [26], which allow here to have estimates of the maximum value of $\left|\widetilde{R}_{\omega}\right|$ on the grid $\mathbb{G}_{q}$. The same remark holds for the next case, using the grid $\mathbb{G}_{2 L q}$.

The use of the same methods for $p>2$ not an even integer is somewhat more delicate: nevertheless, we will prove full $p$-concentration with gap for measurable sets. According to Proposition 48, it would suffice to show $\gamma_{p}^{\star}=1 / 2$ for $p>2$. Essentially, we will do this, but with some necessary modifications. On the other hand we do know $c_{p}^{\star}=1 / 2$ e.g. from the proof of Proposition 33: this proof also provides us a concrete construction, with the product of certain Dirichlet kernels in the proof, which we will make use in some extent. We start with

Lemma 56. Let $p>2$. Then for all $C<1 / 2$, there exists a constant $K:=K_{p}(C)$ with the property that for $q$ large there exists an idempotent $P \in \mathcal{P}_{2 q}$ which satisfies the two inequalities

$$
\begin{aligned}
& \left|P\left(\frac{1}{2 q}\right)\right|^{p}>C \sum_{k=0}^{q-1}\left|P\left(\frac{2 k+1}{2 q}\right)\right|^{p}, \\
& \left|P\left(\frac{1}{2 q}\right)\right|^{p}>K \sum_{k=0}^{q-1}\left|P\left(\frac{k}{q}\right)\right|^{p} .
\end{aligned}
$$

Proof. We use now from Section 5 that for $L$ large enough and $q$ large enough there exists an idempotent in $\mathcal{P}_{q}$, which actually can be taken some Dirichlet kernel $D_{r}$, with say $r:=[q / 4]>c q$ (for some fixed value of $c=c(L, p)<1 / 4)$, such that

$$
\left|D_{r}\left(\frac{1}{2 q}\right)\right|^{L p}>C \sum_{k=0}^{q-1}\left|D_{r}\left(\frac{2 k+1}{2 q}\right)\right|^{L p} .
$$

From now on we fix $L$, so that constants may as well depend on $L$.

Next, we wish to ensure, with some constant $K=K(C, p, L)$, that

$$
\left|D_{r}\left(\frac{1}{2 q}\right)\right|^{L p}>K \sum_{k=0}^{q-1}\left|D_{r}\left(\frac{k}{q}\right)\right|^{L p} .
$$

In view of the concrete form of the Dirichlet kernel, it is obvious, that $\left|D_{r}(1 / 2 q)\right| \geq\left|D_{r}(1 / q)\right|$. Consider now, recalling the estimation of the concentration constants $c_{p}^{\sharp}(q) \rightarrow c_{p}^{\sharp}$ in Section 6, and in particular reflecting back to (47) - (49), the lower estimates

$$
\left|D_{r}\left(\frac{1}{q}\right)\right|^{L p}>\frac{1}{B(L p,[q / 4], q)} \sum_{k=0}^{q-1}\left|D_{r}\left(\frac{k}{q}\right)\right|^{L p} .
$$


As $B(L p,[q / 4], q) \rightarrow B(L p, 1 / 4)>0(q \rightarrow \infty)$, this clearly implies (112).

At this point we proceed as above. First we consider the $L^{\text {th }}$ power of $D_{r}$ and take for $P$ the projected polynomial $M^{-1} \Pi_{2 q}\left(D_{r}^{L}\right)$, with $M:=$ $L r^{L-1}$ a majorant of the Fourier coefficients of $D_{r}^{L}$. The polynomial $P$ may be written as

$$
P=\sum_{k=0}^{2 q-1} \alpha_{k} e_{k}
$$

with all $\alpha_{k} \in[0,1]$ and $\sum \alpha_{k}=P(0)=r / L(\simeq c(L) q)$. So we have

$$
\left|P\left(\frac{1}{2 q}\right)\right|^{p}>C \sum_{k=0}^{q-1}\left|P\left(\frac{2 k+1}{2 q}\right)\right|^{p} .
$$

Moreover, by construction we also have the grid condition

$$
\left|P\left(\frac{1}{2 q}\right)\right|^{p}>K \sum_{k=0}^{q-1}\left|P\left(\frac{k}{q}\right)\right|^{p}
$$

with a certain constant $K=K(C, p, L)$.

Observe that the only required property what $P$ does not have is being an idempotent: here $P \in \mathcal{T}_{2 q} \cap \mathcal{P}^{+}$, while we need some polynomial in $\mathcal{P}_{2 q}$. So we define, as before, a random idempotent $P_{\omega}$ by

$$
P_{\omega}:=\sum_{k=0}^{2 q-1} X_{k}(\omega) e_{k}
$$

where $X_{k}$ are independent Bernoulli random variables, with $X_{k}$ of parameter $\alpha_{k}$, that is, $\mathbb{P}\left(X_{k}=1\right)=\alpha_{k}$. Then again $P(x)=\mathbb{E} P_{\omega}(x)$, and we measure the error done when replacing $P_{\omega}$ by its expectation.

Let us write $X_{k}=\alpha_{k}+\widetilde{X}_{k}$, where $\widetilde{X}_{k}$ is centered and has variance $\alpha_{k}\left(1-\alpha_{k}\right) \leq \alpha_{k}$. So $P_{\omega}(k /(2 q))$ has expectation $P(k /(2 q))$ and variance bounded by $r / L$.

By construction $|P(1 /(2 q))|>K^{1 / p} P(0)>\frac{c K^{1 / p}}{L} q$. So, by Markov Inequality, as before, we find that for $q$ large enough, the inequalities

$$
\left|\frac{P_{\omega}(1 /(2 q))}{P(1 /(2 q))}\right|>1-\varepsilon, \quad\left|\frac{P_{\omega}(1 / q)}{P(1 / q)}\right|>1-\varepsilon
$$

hold with probability $2 / 3$. 
Denoting again $\widetilde{P}_{\omega}(x):=P_{\omega}(x)-P(x)$, let us now consider the sums

$$
\begin{array}{ll}
S(\omega):=\sum_{k=0}^{q-1}\left|P_{\omega}\left(\frac{2 k+1}{2 q}\right)\right|^{p}, & S^{\prime}(\omega):=\sum_{k=0}^{q-1}\left|P_{\omega}\left(\frac{k}{q}\right)\right|^{p}, \\
S:=\sum_{k=0}^{q-1}\left|P\left(\frac{2 k+1}{2 q}\right)\right|^{p}, & S^{\prime}:=\sum_{k=0}^{q-1}\left|P\left(\frac{k}{q}\right)\right|^{p}, \\
\widetilde{S}(\omega):=\sum_{k=0}^{q-1}\left|\widetilde{P}_{\omega}\left(\frac{2 k+1}{2 q}\right)\right|^{p}, & \widetilde{S}^{\prime}(\omega):=\sum_{k=0}^{q-1}\left|\widetilde{P}_{\omega}\left(\frac{k}{q}\right)\right|^{p} .
\end{array}
$$

To compare these again we use the elementary inequality (107) to get $\left|P_{\omega}(k /(2 q))\right|^{p} \leq(1+\varepsilon)|P(k /(2 q))|^{p}+C(\varepsilon)\left|\widetilde{P}_{\omega}(k /(2 q))\right|^{p}$ and thus

$$
S(\omega) \leq(1+\varepsilon) S+C(\varepsilon) \widetilde{S}(\omega), \quad S^{\prime}(\omega) \leq(1+\varepsilon) S^{\prime}+C(\varepsilon) \widetilde{S}^{\prime}(\omega) .
$$

Applying Lemma 54 as before, analogously to (105) we now obtain

$$
\mathbb{E}\left|\widetilde{P}_{\omega}(k /(2 q))\right|^{p} \leq q C_{p}\left(1+\sum \alpha_{k}\right)^{\frac{p}{2}} \leq c^{\prime}(p, L) q^{1-p / 2} .
$$

So for $q$ large enough, similarly to (106), we prove as before that the inequalities

$$
C(\varepsilon) \widetilde{S}(\omega)<\varepsilon S, \quad C(\varepsilon) \widetilde{S}^{\prime}(\omega)<\varepsilon S^{\prime}
$$

hold with probability $2 / 3$, thus combining with the above, we even have

$$
S(\omega)<(1+2 \varepsilon) S, \quad S^{\prime}(\omega)<(1+2 \varepsilon) S^{\prime}
$$

with probability at least $1 / 3$. Taking into account also (113), (114) and (115), we can summarize our estimates so that with positive probability

$$
\begin{gathered}
C S(\omega)<\frac{1+2 \varepsilon}{(1-\varepsilon)^{p}}\left|P_{\omega}\left(\frac{1}{2 q}\right)\right|^{p}, \\
K S^{\prime}(\omega)<\frac{1+2 \varepsilon}{(1-\varepsilon)^{p}}\left|P_{\omega}\left(\frac{1}{2 q}\right)\right|^{p} .
\end{gathered}
$$

Since $\varepsilon$ is arbitrary, we conclude that some $P_{\omega} \in \mathcal{P}_{2 q}$ satisfies the requirements of the Lemma.

At this point, we have all the elements to have the best constant for all $p>1$ not even.

Proposition 57. Let $p>1$ not an even integer. Then there is full p-concentration with gap for measurable sets.

Proof. The proof follows the same lines as the proof of Proposition 48 . We take now $C<1 / 2$ and, instead of choosing $P \in \mathcal{P}_{q}$ satisfying (83) and starting the consruction of $Q$ with that, we start with choosing $P \in \mathcal{P}_{2 q}$ given by Lemma 56, with exponent $2 p>2$.

Note that the only point of the proof of Proposition 48 using the fact that $P$ is in $\mathcal{P}_{q}$ is the grid condition (75), which is given now by (111). 
Thus Lemma 45 applies even in this case, while otherwise the proof is exactly as for Proposition 48 .

\section{REFERENCES}

[1] B. Anderson, J. M. Ash, R. L. Jones, D. G. Rider, B. SAffari, Inégalités sur des sommes d'exponentielles, C. R. Acad. Sci. Paris Ser. I. Math, 296 (1983), 899-902.

[2] B. Anderson, J. M. Ash, R. L. Jones, D. G. Rider, B. Saffari, $L^{p_{-}}$ norm local estimates for exponential sums, C. R. Acad. Sci. Paris Ser. I. Math, 330 (2000), 765-769.

[3] B. Anderson, J. M. Ash, R. L. Jones, D. G. Rider, B. Saffari, Ann. Inst. Fourier, 57 (2007), 1377-1404.

[4] J. M. Ash, Weak restricted and very restricted operators on $L^{2}$, Trans. Amer. Math. Soc., 281 (1984), 675-689.

[5] J. M. AsH, On concentrating idempotents, a survey, Topics in Classical Analysis and Applications in Honor of Daniel Waterman, L. De Carli, K. Kazarian, and M. Milman editors, World Scientific, 2008, 31-44.

[6] J. M. Ash, R. L. Jones, B. SAffari, Inégalités sur des sommes d'exponentielles, C. R: Acad. Sci. Paris Ser. I. Math, 296 (1983), 273-285.

[7] G. F. BAchelis, On the upper and lower majorant properties in $L^{p}(G)$, Quart. J. Math. Oxford (2), 24 (1973), 119-128.

[8] R. P. BoAs, Majorant problems for Fourier series, J. d'Analyse Math., 10 (1962-3), 253-271.

[9] Bonami, A., RÉvész, Sz. Gy., Failure of Wiener's property for positive definite periodic funcztions, C. R. Acad. Sci. Paris Ser I 346 (2008), 39-44.

[10] Bonami, A., RÉvész, Sz. Gy., Concentration of the absolute value integral of idempotents, manuscript.

[11] D. L: Burkholder, Martingale transforms, Ann. Math. Stat., 37 (1966), 1494-1504.

[12] Cowling, M., Some applications of Grothendieck's theory of topological tensor products in harmonic analysis, Math. Ann., 232 (1978), 273-285.

[13] M. Déchamps-Gondim, F. Lust-Piquard, H. Queffélec, Estimations locales de sommes d'exponentielles, C. R: Acad. Sci. Paris Ser. I. Math, 297 (1983), 153-157.

[14] M. DÉchamps-Gondim, F. Lust-Piquard, H. Queffélec, Estimations locales de sommes d'exponentielles, Publ. Math. Orsay 84-01, No.1 (1984), $1-16$.

[15] ERdős, P., RÉNYI, A., On a problem of A. Zygmund, Studies in mathematical analysis and related topics, Stanford University Press, Stanford, California, $1962,110-116$.

[16] J. J. F. Fournier, Majorants and $L^{p}$ norms, Israel J. Math., 18 (1974), $157-166$.

[17] Green, B., Ruzsa, I., On the Hardy-Littlewood majorant problem, Math. Proc. Cambridge Philos. Soc., 137 (2004), no. 3, 511-517.

[18] G. H. Hardy \& J. E. Littlewood, Notes on the theory of series (XIX): a problem concerning majorants of Fourier series, Quart. J. Math. Oxford, 6 (1935), 304-315.

[19] A. E. Ingham, Some trigonometrical inequalities with applications to the theory of series, Math. Zeitschrift, 41 (1936), 367-379.

[20] Khintchine, Einige Sätze über Kettenbrüche, mit Anwendungen auf die Theorie der Diophantischen Approximationen, Math. Ann., 92 (1924), 115-125. 
[21] Komornik, V., Loreti, P., Fourier series in control theory, Springer Monographs in Mathematics. Springer-Verlag, New York, 2005.

[22] Mockenhaupt, G., Bounds in Lebesgue spaces of oscillatory integral operators. Thesis for habilitaton. Siegen: Univ.-GSH Siegen, Fachbereich Mathemtik, (1996), 52 pages.

[23] Mockenhaupt, G., Schlag, W., On the Hardy-Littlewood majorant problem for random sets, arXive:math.CA/0207226v1, for a new version see http://www-math-analysis.ku-eichstaett.de/ gerdm/wilhelm/maj.pdf

[24] H. L. Montgomery, Ten lectures on the interface of number theory and Fourier analysis, Regional Conference Series in Mathematics 84, American Mathematical Society, Providence, 1994.

[25] J. Ortega-Cerdì, J. Saludes, Marcinkiewicz-Zygmund inequalities, Journal of Approximation Theory 145 (2), 237-252 (2007).

[26] R. Salem, A. Zygmund, Trigonometric series whose terms have random signs, Annals of Math., 91 (1954), 245-301.

[27] W. M. Schmidt, Metrical theorems on fractional parts of sequences, Trans. Amer. Math. Soc. 110 (1964), 493-518.

[28] P. Szüsz, Über metrische Theorie der Diophantischen Approximation, Acta Math. Hung. IX (1958), no. 1-2, 177-193.

[29] T. TAO, Integral norms concentration under gap condition, e-mail letter to Szilárd Révész, June 17, 2006.

[30] Tenenbaum, G., Tucsnak, M., Fast and strongly localized observation for the Schrödinger equation, Trans. Amer. Math. Soc., to appear.

[31] Tenenbaum, G., Tucsnak, M., New blow-up rates for fast controls of Schrödinger and heat equations. J. Differential Equations 243 (2007), 70-100.

[32] Turán, P., On a Certain Problem in the Theory of Power Series with Gaps, Studies in mathematical analysis and related topics, Stanford University Press, Stanford, California, 1962, 404-409.

[33] Wiener, N., A class of gap theorems, Ann. Scuola Norm. Sup. Pisa (2) 3 (1934), 367-372.

[34] A. Zygmund, Trigonometric Series, Second edition, I-II, Cambridge University Press, Cambridge, 1959.

(Aline Bonami)

FÉdÉRATION DENIS POISSON

MAPMO-UMR 6628 CNRS

UNIVERSITÉ D'ORLÉANS

45067 ORLÉAns France.

E-mail address: aline.bonami@univ-orleans.fr

(Szilárd Gy. Révész)

A. RÉnyi Institute of Mathematics

Hungarian ACAdemy of Sciences,

Budapest, P.O.B. 127, 1364 Hungary.

E-mail address: revesz@renyi.hu 